\title{
Mid-frontal Theta during Conflict in a Value-based Decision Task
}

\author{
Ulrike Senftleben ${ }^{(1)}$ and Stefan Scherbaum
}

\begin{abstract}
Mid-frontal theta is a sensitive marker for cognitive conflict. However, most research focuses on cognitive control paradigms (e.g., the Flanker task). Here, we ask if mid-frontal theta is also sensitive to response conflicts within value-based decisionmaking. We recorded electroencephalography activity during a value-based binary decision task. In this task, participants collect rewards in a virtual two-dimensional world. In each trial, we present two reward options that are either quick to collect but are smaller in value, or take longer to collect but are larger in value. The subjective value of each option is driven by the options' value and how quickly they can be reached. We used this task to investigate three types of potential conflicts: choice ambiguity, choice repetitions, and temporal delay. We investigated choice
\end{abstract}

\section{INTRODUCTION}

In value-based decision-making, people decide between different options based on subjective values that they associate with each option (Rangel, Camerer, \& Montague, 2008). Value-based decisions are often multi-attribute decisions with potential cost-benefit trade-offs, for example, when deciding if you want to snooze your alarm in the morning for 10 more minutes of sleep but risk running late as a consequence. The values associated with each option (here: snooze the alarm or get up) are very subjective, and there is no objective "right" or "wrong" answer. Rather, the subjective values of all choice options are calculated and compared in order to make a final choice (Pisauro, Fouragnan, Retzler, \& Philiastides, 2017; Rodriguez, Turner, \& McClure, 2014; Kable \& Glimcher, 2007). This comparison of options rests on competition between them, which induces decision conflict (Lin, Saunders, Hutcherson, \& Inzlicht, 2018; Shenhav \& Buckner, 2014; Jocham, Hunt, Near, \& Behrens, 2012).

This aspect - the role of decision conflict - of valuebased decisions is often overlooked (but see Cheng \& González-Vallejo, 2018), and hence, we do not know much about how this conflict is solved. Rather, most of the insights into conflict come from research on objective conflicts in inhibitory control tasks. Inhibitory control tasks, such as the Flanker (Eriksen \& Eriksen, 1974), Stroop

Technische Universität Dresden, Germany repetition by biasing participants toward one option for two trials and then testing how that affects the subsequent decision. We manipulated choice ambiguity by varying the subjective values of the decision options, and temporal delay by making one option quick to collect and one longer to collect. The behavioral results showed the expected effects: Decision times were shorter for unambiguous choices, participants showed a tendency to repeat the previous choice and decision times were shorter for repetitions, and decision times were shorter for earlier available choices. Response-locked mid-frontal theta power was increased for choice switches as compared to choice repetitions, and for the later available as compared to the earlier available option, but we found no effect of ambiguity.

(Stroop, 1935), or Simon task (Simon \& Rudell, 1967), require the inhibition of a prepotent response or distractor information in order to make a correct response. For example, in the Flanker task (Eriksen \& Eriksen, 1974), participants are asked to indicate if an arrow is pointing to the left or the right. The arrow is surrounded by distractor arrows that can either point in the same direction as the target arrow (congruent trial), or that can point in the opposite direction as the target arrow (incongruent trial). In congruent trials, competition (and therefore conflict) is low because both the target and the distractor information elicit the same response. In incongruent trials, however, competition (and therefore conflict) is high because the target and distractor information elicit different responses. This conflict between alternative response options is overcome by competitive mechanisms that lead the system to finally settle for one option (Cohen, Dunbar, \& McClelland, 1990). To improve performance in future response, it has been proposed that the conflict also must be detected and that a conflict monitoring mechanism picks up on this detected conflict and recruits cognitive control resources (Botvinick, Cohen, \& Carter, 2004; Botvinick, Braver, Barch, Carter, \& Cohen, 2001). On a neural level, it has been found that conflict is reliably accompanied by increased power in mid-frontal theta oscillations $(4-7 \mathrm{~Hz}$ ) and has been proposed to reflect the conflict detection mechanism. Mid-frontal theta power has been shown to track conflict in a variety of inhibitory control tasks, such 
as the Flanker task (Nigbur, Ivanova, \& Stürmer, 2011), the Stroop (Zhao et al., 2015; Kovacevic et al., 2012; Hanslmayr et al., 2008), and the Simon task (Rosen, Padovan, \& Marinkovic, 2016; Cohen \& Donner, 2013; Nigbur et al., 2011). Furthermore, mid-frontal theta is also associated with the reduction of switch costs in task switching (Cooper et al., 2019). Evidence from source reconstruction, intracranial recordings, and functional neuroimaging studies indicates that this signal originates in the dorsal anterior cingulate cortex (dACC) and surrounding medial prefrontal structures (Zavala et al., 2018; Töllner et al., 2017; Cavanagh, Figueroa, Cohen, \& Frank, 2012; Kovacevic et al., 2012; Womelsdorf, Vinck, Leung, \& Everling, 2010; Cohen, Ridderinkhof, Haupt, Elger, \& Fell, 2008). The dACC has been suggested to play a key role in monitoring and detecting conflict (e.g., Botvinick et al., 2001, 2004; Yeung, Botvinick, \& Cohen, 2004). Indeed, mid-frontal theta power has been suggested to be a predominant mechanism for cognitive or inhibitory control (Verguts, 2017; Cavanagh \& Frank, 2014). Theta oscillations are a prime candidate for information exchange between neuronal populations because they can organize and synchronize higher-frequency activity. For example, recordings from macaques and rats have shown that theta and gamma frequencies interact with each other, a process called cross-frequency coupling, so that theta phase is coupled with gamma amplitude (Voloh, Valiante, Everling, \& Womelsdorf, 2015; Lisman \& Jensen, 2013; Tort, Komorowski, Manns, Kopell, \& Eichenbaum, 2009; Canolty et al., 2006). Hence, theta is able to modulate gamma activity and therefore to implement top-down cognitive control processes, which in turn produce the typical effects of slower RTs in incongruent trials and posterror or postconflict adjustments of RTs (Verguts, 2017).

Whereas the involvement of mid-frontal theta in inhibitory control and conflict processing has been studied extensively, almost no research has considered conflict in other tasks. A notable exception is a recent study by Lin et al. (2018), who investigated mid-frontal theta power and pupil dilation in an intertemporal choice task (the choice between a smaller, but sooner available reward and a larger, but delayed reward; e.g., $15 €$ now or $20 €$ in 2 weeks). They showed that theta tracked subjective conflict: Decisions where both choice options were equally valuable (ambiguous decision) were accompanied by higher theta power than decisions where one choice option was more valuable (unambiguous decisions). Here, we build on this perspective and ask if mid-frontal theta tracks different types of conflict in value-based decision-making. Therefore, we strive to gain more insights into the role of mid-frontal theta in conflict processing, and into potential differences or similarities between conflict in subjective value-based decisions and in objective inhibitory control tasks.

As mentioned above, value-based decision tasks do not induce conflict in the same way as classical inhibitory control tasks; there is no distractor information or prepotent response that induces conflict and that needs to be suppressed. At the same time, however, all decision options need to be assessed in order to generate subjective values and make a final choice. Hence, in this regard, all choice options compete against each other for selection. Therefore, value-based choices always include conflict between response options and, interestingly, the proposed competition processes to resolve this conflict bear strong similarities to the process proposed for inhibitory control tasks (Glöckner, Hilbig, \& Jekel, 2014; Glöckner \& Pachur, 2012). Here, we investigate this conflict in more detail in a value-based decision game. In this decision game, participants collect rewards in a two-dimensional virtual world (Schoemann \& Scherbaum, 2020; Senftleben et al., 2019; Scherbaum et al., 2016). In this virtual world, two rewards appear in each trial: One reward can be collected quickly (small/near [SN] option), but is worth less, whereas the other reward takes longer to collect, but is worth more (large/far [LF] option).

We use this task to probe three potential sources of conflict: conflict induced by choice ambiguity, conflict induced by the choice in previous trials, and conflict induced by choice attributes. All three types of conflict can be derived from neural network models as they have been proposed as mechanisms for conflict resolution (Glöckner et al., 2014; Jones, Cho, Nystrom, Cohen, \& Braver, 2002; Servan-Schreiber, Printz, \& Cohen, 1990; please see Appendix A for a formalized derivation). The first source of conflict is choice ambiguity. Choice ambiguity is defined by how (dis)similar two choice options are: If one option is clearly more valuable than the other option, conflict is low and the decision is unambiguous. In turn, if both options are equal in subjective value, conflict is high and the decision is ambiguous. Hence, ambiguous decisions are associated with increased decision times (Lin et al., 2018; Monterosso et al., 2007), as well as with increased conflict as indexed by higher mid-frontal theta power (Lin et al., 2018) and increased BOLD activity in the dACC (Marco-Pallarés, Mohammadi, Samii, \& Münte, 2010). Therefore, we expect higher mid-frontal theta power and longer decision times in ambiguous choices.

The second source of conflict lies in the choice repetition effect, the tendency to repeat choices. This choice repetition tendency emerges because of residual activity from the previous trial, which creates a bias toward the previously chosen option (Schoemann \& Scherbaum, 2020; Senftleben et al., 2019; Scherbaum et al., 2016). Hence, conflict is low when repeating the same decision and conflict is high when switching to an alternate decision. Therefore, we expect higher mid-frontal theta power and longer decision times in choice switches as compared to choice repetitions.

The final source of conflict is the temporal delay of the choices. In our decision game, one option - the SN optionis always closer and therefore can be collected quicker (comparable to the smaller/sooner and larger/delayed 
rewards in intertemporal choice). Previous research has shown that humans perceive time in a nonlinear way (Kim \& Zauberman, 2009; Zauberman, Kim, Malkoc, \& Bettman, 2009), and that information on temporal attributes is accumulated at a quicker rate than information on monetary or value attributes (Scherbaum, Dshemuchadse, \& Goschke, 2012). These mechanisms have been shown to create a bias toward options that are available earlier in time (Scherbaum et al., 2012). Therefore, we expect conflict to be lower when choosing the earlier available option (SN option) and we expect conflict to be increased when choosing the option that is later available (LF option), which should correspond to higher mid-frontal theta power and longer decision times for the LF option.

\section{METHODS}

\section{Data Statement}

We report how we determined our sample size, all data exclusions (if any), all manipulations, and all measures in the study. All data and analysis scripts are openly accessible at https://osf.io/bphx9/.

\section{Participants}

Data collection for the study was combined with data collection for a task switching study because of economic reasons. We performed a power-based sample size calculation for the choice repetition effect, because this was the only effect we tested in a previous study and for which we had a specific effect size estimate. Based on this effect size (differences in decision times: $g=0.39$ ), we needed 73 participants in order to achieve a power of 95\%. We recruited 81 participants via the ORSEE-based database of the Department of Psychology of the TU Dresden, Germany. The mean age was 24.22 years $(S D=$ 6.38 years, range $=18-57$ years $) ; 58$ participants were female. Fourteen participants did not complete our experiment because their decision-making did not allow for our experimental manipulation. Of the remaining 67 participants, 47 were female and the mean age was 23.88 years $(S D=5.00$ years, range $=18-39$ years $)$. One additional participant was excluded for EEG analysis because of poor EEG data quality (final EEG sample: $n=66,46$ female, mean age $=23.94$ years, $S D=5.01$ years, range $=18-19$ years $)$. All participants had normal or corrected-to-normal vision and color vision. Because of exclusion criteria for the task switching study (which used flickering stimuli), we only included participants who had no family history of epilepsy or seizures. Participants gave informed consent and received reimbursement of $8 €$ per hour or class credit, as well as the money they collected within the delay discounting task. The experiment was approved by the local ethics committee and was conducted in accordance with the Declaration of Helsinki.

\section{Apparatus and Stimuli}

Participants performed the task in an EEG lab that was sound-attenuated and electrically shielded. They were seated approximately $60 \mathrm{~cm}$ in front of the screen, with their heads placed on a chin rest. The task was presented on a 17-in. screen (resolution of $1280 \times 1024$ px, $72-\mathrm{Hz}$ refresh rate). The experiment was controlled using the Psychtoolbox Version 3 (Kleiner et al., 2007; Brainard, 1997) in MATLAB 2006b on a Windows XP personal computer. Participants controlled a Logitech USB mouse to make their responses. The decision game consisted of a two-dimensional world of $20 \times 20$ fields, with one field consisting of $50 \times 50$ pixels (see Figure 1 ). The stimuli used within this paradigm were circular coin shapes with a diameter of 50 pixels. The avatar that participants controlled was a red circle that could be moved freely from field to field by clicking with the computer mouse into either vertical or horizontal adjacent fields outlined in white. The coins used as reward stimuli were gold with the value written inside the coin in red. Throughout the whole task, the remaining time within each block was displayed above the avatar.

\section{Procedure}

As the study was conducted together with a task switching study, the order of both tasks was balanced across

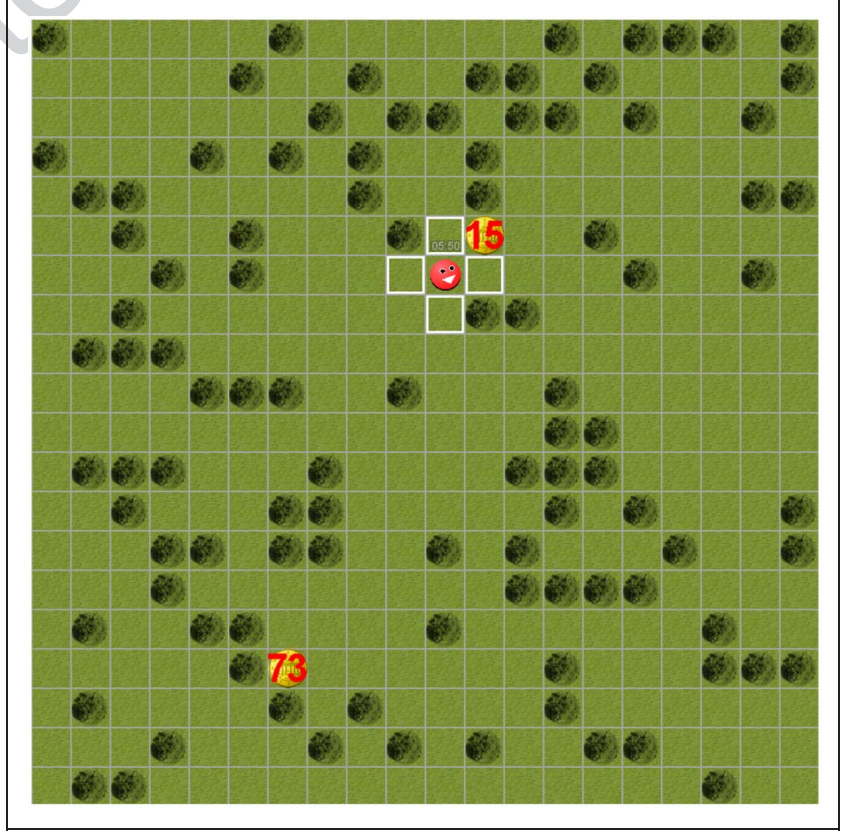

Figure 1. Decision game. Participants controlled the red avatar by using the computer mouse and clicking into vertical or horizontal fields (outlined in white). Throughout the whole task, the remaining time within the block was displayed above the avatar. In each trial, two coins appeared. Once the avatar reached one coin, both coins disappeared, the accumulated credit was displayed below the avatar, and the avatar could not be moved until the next trial started with the appearance of two new coins. Dark green fields (representing trees) were included for better spatial orientation; they did not obstruct movement and could be crossed freely. 
participants. Here, we will only focus on the decision-making task. Before the experiment, participants completed a vision test to check for normal or corrected-to-normal vision as well as color vision. To minimize EEG artifacts, participants were instructed to remain in a relaxed and still position throughout the task. Before starting the task, the EEG signal was shown to the participants and common artifacts were demonstrated (i.e., eye blinks, lateral eye movements, muscle tension in shoulders, jaw and neck) to emphasize the importance of a relaxed, still position. Participants practiced the task for 2 min before the experiment started.

The decision-making task itself consisted of five blocks of $8 \mathrm{~min}$. In between the five blocks of the game, participants had to wait for at least $15 \mathrm{sec}$ before they could continue. Within the decision game, participants made decisions between two options in a trial-wise fashion. In each trial, two coins appeared at the same time. Participants had to choose between these two coins by moving the avatar field by field until it reached the chosen coin. Note that coins were positioned at an angle of at least $90^{\circ}$ to each other with respect to the avatar, so that moving toward one coin increased the distance to the other one. Upon reaching that coin, both coins disappeared and the accumulated credit collected so far appeared (one credit equaled 0.01 cent). The next trial started with the appearance of two new coins. The intertrial interval (ITI) was either $600 \mathrm{msec}$ or $1500 \mathrm{msec}$ (see Task Design section). During the ITI, the mouse cursor was locked to the position of the avatar; it could only be moved again when the next trial started and new coins appeared.

\section{Task Design}

The study had a within-subject design in which participants performed the decision task in two different parts: a measurement part intended to measure participants' individual decision-making (the first block of $8 \mathrm{~min}$ ) and an experimental part intended to capture all three conflict types (four blocks of $8 \mathrm{~min}$ ). For both parts, trials were constructed so that one coin was closer to the avatar but had a smaller value (SN option), and the other coin was further away but had a larger value (LF option).

The measurement part of the task consisted of one block of trials with a wide range of combinations of distances and values. The SN option was either two or three fields away from the avatar; the LF option was either 1, 4, 8, or 12 fields further away from the avatar than the SN option. In each trial, the value of the LF option was drawn randomly from a range of $65-85$ credits, whereas the value of the SN option randomly varied between $20 \%$ and $95 \%$ of the LF option. During the measurement block, the ITI was kept constant at $600 \mathrm{msec}$.

We used the choice data of this measurement block to estimate participants' indifference points. Indifference points describe the specific ratio of SN to LF value where both options are equally attractive to the participant (i.e., the probability of choosing either the LF or SN option is $50 \%$, respectively). For each participant, we calculated indifference points for each distance (1, 4, 8, 12 fields) by fitting a logistic function to the choice data and taking the point of inflection (for details, see Appendix B). Based on these four indifference points for the four distances used in the measurement block, we interpolated the indifference points for the remaining distances between 1 and 12 . We used either a linear, hyperbolic, or exponential model for interpolation based on the best fit with the four available indifference. ${ }^{1}$ As a result, we had estimates of indifference points for each distance between 1 and 12, which we then used to construct trials in the subsequent experimental part. If participants discounted very highly or not all (i.e., they always chose the SN or always chose the LF option), the experiment ended after the measurement block because the manipulation of the experimental blocks would not have been possible.

The experimental part consisted of four blocks of $8 \mathrm{~min}$. We built mini-blocks of three trials where the first two trials served as bias trials (SN or LF) and the third trial as target trials (SN or LF or neutral; see Figure 2). For the bias trials, we chose reward values so that participants were more likely to choose either the SN option or the LF option, respectively. We achieved this by setting the value of the SN option either to 30\% above the indifference point, making the $\mathrm{SN}$ option more attractive, or by setting the value of the LF option to $30 \%$ below the indifference point, making the LF option more attractive. Based on participants' indifference points, we identified for which distances this manipulation was possible (as the indifference point had to be either below $70 \%$ for attractive SN trials and above 30\% for attractive LF trials) and drew randomly from these distances for each bias trial. The value of the LF option varied between 65 and 85 .

We constructed three types of target trials: $\mathrm{SN}, \mathrm{LF}$, and neutral. SN and LF target trials were set up as described above for SN and LF bias trials. For neutral target trials, we set the value of the SN option to the indifference point, so that the SN and LF option were equally attractive to the participant. Distances for neutral target trials were drawn randomly from a range of 1-12 fields. We also varied the ITI between trials. We used a short ITI (600 msec) and a long ITI (1500 msec). The ITI between the second bias trial and the target trial was always short, in order to maximize the effect of the bias. For the other two ITIs (target trial to first bias trial; first bias trial to second bias trial), one was short and one was long, but the order was randomized in order to keep the sequential structure of the trials from becoming too obvious.

In summary, our task design allowed us to tailor the choice options to each participant's individual choice preferences in order to test the three potential sources of conflict. For choice ambiguity, we compared neutral target choices (highly ambiguous) with SN and LF target trials (unambiguous). For the choice repetition effect, we compared neutral target choices where participants 
Figure 2. Task design in the experimental part. Bias trials were either SN or LF trials, and both bias trials in a mini-block were the same trial type. Target trials were $\mathrm{SN}, \mathrm{LF}$, or neutral trials. The design was fully balanced, resulting in six different mini-block scenarios (2 bias types $[\mathrm{SN}, \mathrm{LF}] \times 3$ target types [SN, LF, neutral]).

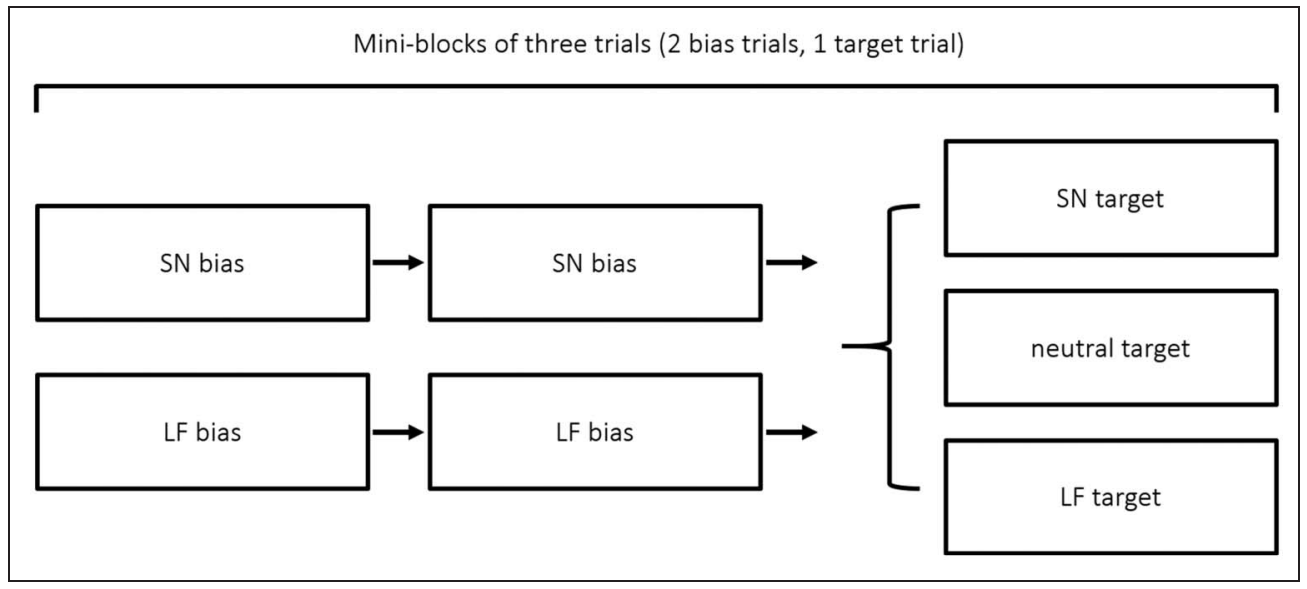

repeated versus switched from their choice in the bias trial. Finally, for temporal delay, we compared SN (available sooner) with LF target choices (available later).

\section{EEG Recording and Preprocessing}

We recorded continuous scalp EEG from $64 \mathrm{Ag} / \mathrm{AgCl}$ electrodes at a sampling rate of $500 \mathrm{~Hz}$, using VisionRecorder software and BrainAmp amplifiers (Brain Products). Placement of the electrodes was in accordance to the International 10-20 system using EasyCap equipment (EasyCap). The EEG setup included one electrooculogram electrode underneath each eye, the scalp electrode AFz was used as ground, and the left mastoid electrode was used as reference during recording. Impedances were kept below $5 \mathrm{k} \Omega$.

Data preprocessing was carried out using EEGLAB 2019.0 (Delorme \& Makeig, 2004) running in MATLAB R2015a. First, we filtered the raw EEG signal (1-Hz highpass filter, 70-Hz low-pass filter, 45- to 55-Hz notch filter). We resampled the data to $250 \mathrm{~Hz}$ and rereferenced to linked mastoids. We initially epoched data around the stimulus onset $(-2$ to $5 \mathrm{sec}$ ) in order to preserve the prestimulus baseline. Epochs were visually inspected to reject bad epochs. We removed 2.82 epochs on average ( $S D=$ 5.32 epochs). Next, we applied independent component analysis (ICA) as implemented in EEGLAB on a shorter time window of interest ( -700 to $3000 \mathrm{msec}$ ) in order to reduce the amount of overlapping data between trials and therefore increase ICA quality. We then applied the resulting ICA weights to the original full-length epochs and removed components containing eye or muscle artifacts. On average, we removed 3.39 components $(S D=$ 1.41 components). After this, we re-epoched the data from -2 to $1.5 \mathrm{sec}$ around the response (first click of the computer mouse indicating the decision).

\section{Time-Frequency Analysis}

Time-frequency analyses were carried out in MATLAB using custom-written scripts and followed common analysis steps described in the literature (e.g., Cohen \& Donner,
2013; Cavanagh, Figueroa, et al., 2012; Cohen \& Cavanagh, 2011). We performed time-frequency decomposition on a single-trial basis by multiplying the power spectrum of the EEG signal by the power spectrum of complex Morlet wavelets and then taking the inverse fast Fourier transform. Complex Morlet wavelets were defined as $e^{i 2 \pi t f} e^{-t^{2} /\left(2 \sigma^{2}\right)}$, where $t$ is time, $f$ is frequency, and $\sigma$ is the width of the wavelet defined as $n /(2 \pi f)$. We extracted 40 frequencies in 40 logarithmically spaced steps from 1 to $40 \mathrm{~Hz}$. The number of cycles, $n$, increased logarithmically with each frequency from three cycles at $1 \mathrm{~Hz}$ to eight cycles at $40 \mathrm{~Hz}$. From the resulting complex signal, we obtained power values at each time point and each frequency by taking the squared magnitude of the result of the convolution $Z$ (real $[z(t)]^{2}+\operatorname{imag}[z(t)]^{2}$ ). We then normalized power via a decibel $(\mathrm{dB})$ transform in each trial to a baseline period during the ITI $(-400$ to $-200 \mathrm{msec}$ before stimulus onset $)$ : $\mathrm{dB}$ power $=10 \times \log 10($ power/baseline $)$. We were interested in three comparisons in order to probe the three conflict types: unambiguous versus ambiguous target trials, choice repetitions versus switches in neutral target trials, and SN versus LF targets. For statistical analysis, we determined a time window of interest of -800 to $400 \mathrm{msec}$ around the response. Based on the literature, we focused on electrode $\mathrm{FCz}$, where conflict-related theta power is typically largest (Lin et al., 2018; Cohen \& Donner, 2013; Cavanagh, Figueroa, et al., 2012; Cavanagh, ZambranoVazquez, \& Allen, 2012; Nigbur et al., 2011). We calculated average theta power $(4-7 \mathrm{~Hz})$ for electrode $\mathrm{FCz}$ at each sample point within the time window of interest, separately for each condition and participant. We were interested in identifying time clusters where average theta power differed significantly between conditions. For each comparison, we computed a $t$ test at each sample point in order to identify significant time clusters. In order to correct for multiple comparisons, we used permutation testing to identify the appropriate cluster size threshold for $\alpha=$ .05. Specifically, we built up a null distribution of temporal cluster size by shuffling each participant's trials so that trials were randomly allocated to either condition of interest. We then computed $t$ tests at each sample point and saved the maximal cluster size (i.e., largest cluster of continuous 
significant $t$ tests). We repeated this process for 10,000 iterations in order to build up the null distribution. We determined the $95 \%$ percentile threshold of this null distribution, which then yielded the (multiple-comparisoncorrected) cluster size threshold for our empirical data analysis. This threshold was 62 samples (= $248 \mathrm{msec})$ for the comparison of ambiguous versus unambiguous target trials, 59 samples $(=236 \mathrm{msec})$ for repetitions versus switches, and 60 samples (=240 msec) for the comparison of SN versus LF targets. We then compared the empirical cluster size with this null distribution in order to compute the corrected $p$ values.

\section{RESULTS}

\section{Behavioral Results}

The results of the measurement block and further information on the experimental block are summarized in Appendix B. Here, we focus on the experimental block data. All data processing was carried out in MATLAB
R2015a, and additional statistical analyses were carried out in JASP 0.11.1 (JASP Team, 2019).

First, we analyzed participants' choice behavior. In the experimental block, we had implemented experimental manipulations to test our hypotheses: We manipulated choice preference (creating unambiguous SN and LF targets, and ambiguous or neutral trials), and we manipulated choice sequences in order to measure choice repetition. Specifically, we expected participants to choose according to the trial type (i.e., preferring $\mathrm{SN}$ in $\mathrm{SN}$ trials, LF in LF trials [unambiguous trials], and having equal percentages of $\mathrm{SN}$ and LF choices in neutral trials [ambiguous trials]). We further expected participants to show a higher percentage of LF choices after LF bias trials and a lower percentage of LF choices after SN bias trials, because of choice repetition. We tested this with a $3 \times 2$ factorial repeated-measures ANOVA with the factors target type (SN, LF, neutral) and bias type (SN, LF). The dependent variable was the percentage of $\mathrm{LF}$ choices in target trials; we only included trials with successful bias trials (i.e., where in the second bias trial of the mini-block, participants
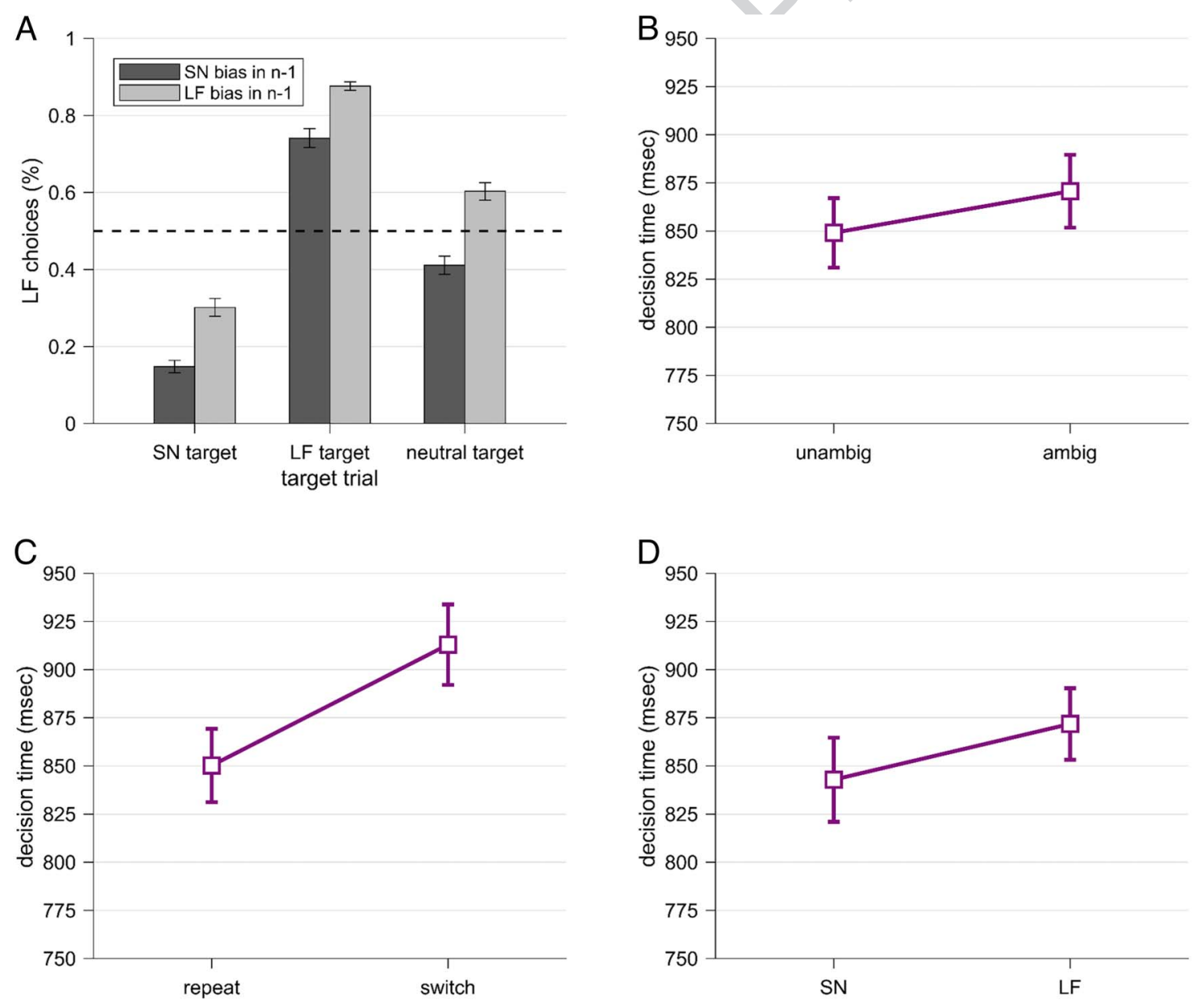

Figure 3. Behavioral results. (A) Percentage of LF choices for each target trial type: unambiguous SN, unambiguous LF, and ambiguous neutral targets. Dark (light) gray bars represent choices after successful SN (LF) bias trials. (B) Decision times for unambiguous target trials (SN/LF) and ambiguous target trials (neutral). (C) Decision times for choice repetitions and choice switches in ambiguous target trials (after successful bias trials). (D) Decision times for SN target choices and LF target choices. Error bars represent standard errors. 
choice was consistent with the bias type (SN choice in $\mathrm{SN}$ bias trial, LF choice in LF bias trial)). As expected, LF choice percentages differed significantly between the different target types, $F(2,132)=545.38, p<.001, \eta^{2}=.62$. As Figure 3A shows, LF choice percentages were low in SN target trials, high in LF target trials, and around 50\% in neutral/ambiguous target trials. Post hoc tests revealed that all target types differed significantly from each other, all $p s<$ .001 (Holm-corrected). Hence, our choice manipulation based on subjective values worked just as expected. We also found a significant influence of bias type, $F(1,66)=$ 138.91, $p<.001, \eta^{2}=.07$. As Figure 3 shows, LF choice percentages were lower after SN bias trials and higher after LF bias trials, as supported by post hoc tests, $p<$ .001 (Holm-corrected), $d=1.44$. This is in line with our expectations that choice repetition effects would impact target trial choices. Furthermore, this effect was present in the vast majority of subjects (see Appendix B for more details). The interaction between bias type and trial type was not significant, $F(2,132)=2.46, p=.09, \eta^{2}=.001$. In summary, our choice manipulation was successful and we found evidence for choice repetition effects.

Next, we analyzed participants' decision times. Specifically, we expected to find effects of choice ambiguity, of choice repetition, and temporal delay. For the choice ambiguity effect, we hypothesized that neutral targets were more ambiguous and therefore induced more conflict as compared to LF or SN choices (unambiguous), which should lead to longer decision times for neutral choices. We tested this hypothesis with a one-tailed $t$ test. As expected, decision times were significantly longer for ambiguous target choices, $t(66)=3.99, p<.001, g=$ 0.14 (ambiguous: $M=870.69 \mathrm{msec}, S D=18.90 \mathrm{msec}$; unambiguous: $M=849.04 \mathrm{msec}, S D=18.03 \mathrm{msec}$ ).

For the choice repetition effect, we expected faster decision times for repetitions than for switches. To test this, we analyzed decision times in ambiguous target trials after successful bias trials. We only focused on ambiguous target trials to separate the effect of choice repetition from the effect of choice ambiguity and choice type. As expected, decision times in ambiguous target trials were significantly slower when participants repeated the choice from the bias trial versus switching to the alternative choice, $t(66)=4.64$, $p<.001, g=0.38$ (one-tailed; repetitions: $M=850.22 \mathrm{msec}$, $S D=19.03$ msec; switches: $M=912.93 \mathrm{msec}, S D=$ 20.89 msec). Finally, for the temporal delay effect, we expected decision times in SN target choices to be shorter than in LF target choices. As expected, decision times were significantly shorter for SN target choices, $t(66)=1.87, p=$ $.03, g=0.17$ (one-tailed; SN: $M=842.83 \mathrm{msec}, S D=$ $21.86 \mathrm{msec}$ LF: $M=871.79 \mathrm{msec}, S D=18.57 \mathrm{msec})$. In summary, our behavioral results indicate that participants showed the expected choice ambiguity, choice repetition, and temporal delay effects.

\section{Mid-frontal Theta Power}

We hypothesized that choice ambiguity, choice repetition, and temporal delay should each induce conflict and should therefore elicit increased mid-frontal theta
Figure 4. Time-frequency representations and mean theta power for the choice ambiguity effects. All plots are responselocked (0 msec corresponds to the first response). There were no significant differences in theta power between ambiguous choices (neutral targets) and unambiguous choices (SN and LF targets). (A) and (B) show dB-converted changes in power as compared to baseline ( -400 to $-200 \mathrm{msec}$ before stimulus onset) for ambiguous (ambig) and unambiguous (unambig) choices. (C) Averaged theta power $(4-7 \mathrm{~Hz})$ for both conditions. Error areas represent standard errors. (D) Topographies for the difference between mean theta power in switches and repetitions, averaged across two time windows.

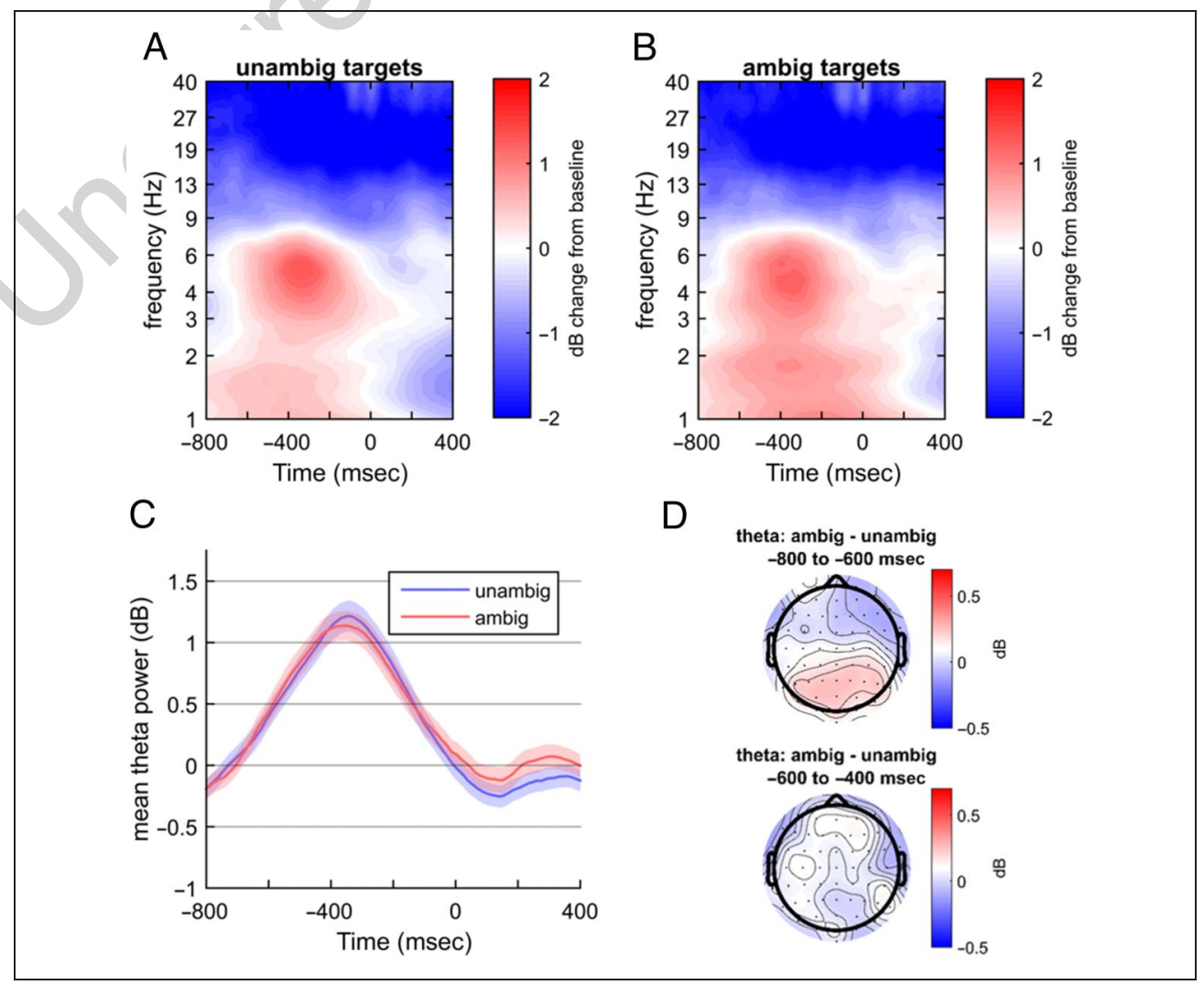


Figure 5. Time-frequency analysis results of choice repetitions and choice switches in ambiguous target trials. All plots are response-locked (0 msec corresponds to the first response). (A) and (B) show dB-converted changes in power as compared to baseline $(-400$ to $-200 \mathrm{msec}$ before stimulus onset) for choice repetitions and switches. (C) Averaged theta power $(4-7 \mathrm{~Hz})$ for repetitions (rep) and switches (sw). Significant time clusters of differences are marked in gray. Error areas represent standard errors. (D) Topographies for the difference between mean theta power in switches and repetitions, averaged across two time windows.
Figure 6. Time-frequency analysis results of SN targets and LF targets. All plots are response-locked $(0 \mathrm{msec}$ corresponds to the first response). (A) and (B) show dB-converted changes in power as compared to baseline $(-400$ to -200 msec before stimulus onset) for SN and LF targets. (C) Averaged theta power (4-7 Hz) for SN and LF targets. Significant time clusters of differences are marked in gray. Error areas represent standard errors. (D) Topographies for the difference between mean theta power in LF and SN targets, averaged across two time windows.
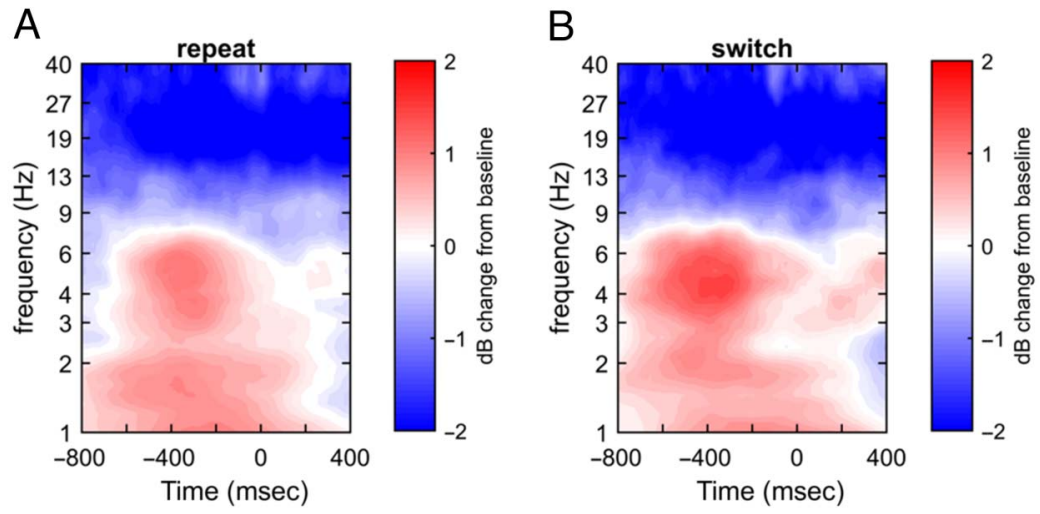

C
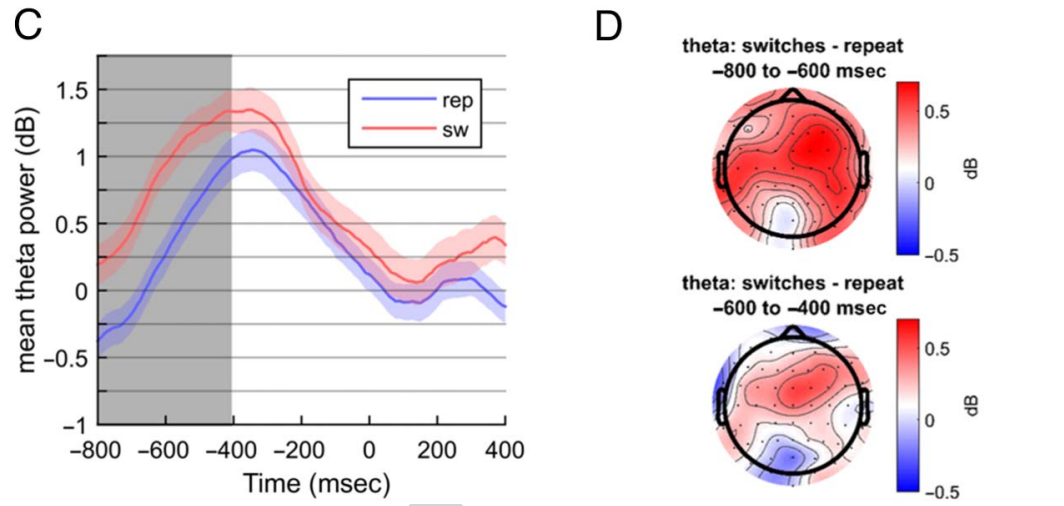

C

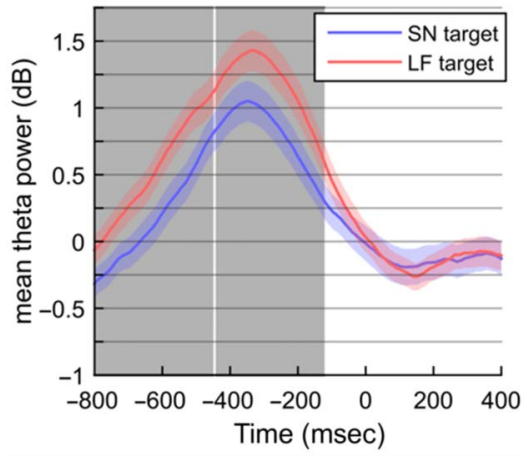

B
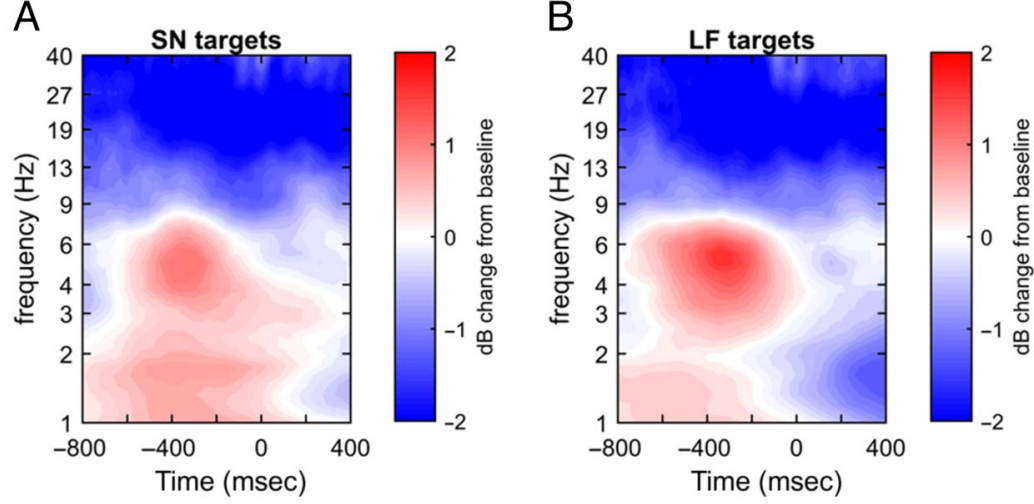

D

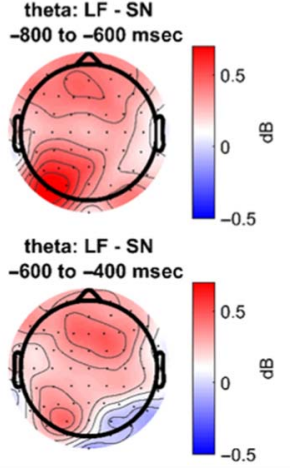


power. We tested this by comparing response-locked theta power at electrode FCz. We compared the same conditions as in the decision time analysis: 1) unambiguous (SN/LF) versus ambiguous (neutral) target trials, 2) choice repetitions versus choice switches in ambiguous target trials, and 3) SN versus LF target choices.

As with the behavioral analysis, we only included trials with successful bias trials. We averaged theta power $(4-7 \mathrm{~Hz})$ and performed consecutive $t$ tests in order to test for significant time clusters (corrected for multiple comparisons based on permutation testing; see Methods section for a detailed description). Against our hypotheses, we did not find any significant differences in mid-frontal theta power between neutral and SN/LF target trials (see Figure 4). For the choice repetition effect, we found a significant time cluster where theta power was higher for switches than for repetitions, as expected: -800 to $-408 \mathrm{msec}, p=.013$ (see Figure 5). Finally, for the comparison of LF and SN target choice, we found two significant time clusters where theta power was higher for LF than for SN, as expected: -800 to $-452 \mathrm{msec}$, $p=.02$, and -440 to $-124 \mathrm{msec}, p=.026$ (see Figure 6). As an additional exploratory analysis, we also included all of these effects as regressors in a single-trial multiple regression in order to gain more insights into the time-frequency profiles of these effects (see Appendix B, Figure A6).

\section{DISCUSSION}

In this study, we investigated three potential sources of conflict in value-based decision-making: choice ambiguity, choice repetition, and temporal delay. We analyzed conflict measures on a behavioral level in the form of choices and decision times, and on a neural level in the form of mid-frontal theta power. We found the expected behavioral effects for all three types of conflict, and we found conflict effects on a neural level for choice repetition and for temporal delay, but not for choice ambiguity.

\section{Conflict in Value-based Decision-Making}

Conflict, on a neural level, can be understood as competition between different responses or representations (Jones et al., 2002). These different responses can be objectively correct or incorrect as, for example, in an incongruent Flanker trial, there is competition between the correct target response (e.g., left) and the incorrect distractor response (e.g., right); or in the Stroop task, there is competition between the color of the word (e.g., red) and the meaning of the word (e.g., "green"). However, this objective type of conflict is not the only type of conflict that plays a role in our decision-making and behavior. In value-based decision-making, the competing responses represent choice options with their individual subjective value. Hence, one could call these conflicts subjective conflicts. Here, we investigated such subjective conflicts in value-based decisions in detail by focusing on three potential sources of such conflicts, namely, choice ambiguity, choice repetition, and temporal delay. Our results confirmed that all three of these sources induce longer decision times, which usually is a sign of conflict, as we will now discuss.

First, we expected to see more conflict in ambiguous choices. Choice ambiguity is low when one decision option is clearly higher in value, whereas ambiguity is high when both decision options have equal value. Previous research has shown that ambiguity causes increased conflict, as indicated by increased decision times and stronger competition between the decision options (Cheng \& González-Vallejo, 2018; Lin et al., 2018; O’Hora, Carey, Kervick, Crowley, \& Dabrowski, 2016; Scherbaum et al., 2016; Scherbaum, Dshemuchadse, Leiberg, \& Goschke, 2013; Monterosso et al., 2007). We replicated this effect on a behavioral level, which showed that participants were sensitive to the ambiguity of the choice scenarios: They preferred the clearly more valuable option in unambiguous trials (i.e., LF option in LF trials, SN option in SN trials) and showed no clear preference in ambiguous trials. Furthermore, as expected, decision times in ambiguous trials were slower than decision times in unambiguous trials. Overall, this fits well with previous research showing that similar choice options make it more difficult to decide because of increased competition between the options. Hence, we expected that this should also be reflected in increased mid-frontal theta power for ambiguous choices. However, this prediction was not supported by our data: We found no evidence for any difference in theta power between ambiguous and unambiguous choices. This is surprising, as Lin et al. (2018) found effects of choice ambiguity on midfrontal theta power in an intertemporal choice task (where participants have to choose between a sooner available, smaller reward and a later available, larger reward). In their task, choice ambiguity was manipulated in a parametric manner based on subjective values, similar to our study. Mid-frontal theta tracked this ambiguity so that theta power was high for ambiguous and low for unambiguous choices. Hence, we must speculate about the reasons for our null effect, which leads us to two potential explanations on two levels. The first explanation on a technical level concerns our choice ambiguity manipulation, which was based on subjective preference estimates. There are several potential issues to consider. First, the subjective preference estimates contained noise. Whereas, on average, participants' choice percentages in the experimental block validate our estimates from the measurement block, there was substantial interindividual variance and choice preference in the experimental part diverged from the theoretical preference estimated in the measurement block (see Figure A4). This directly affects our choice ambiguity analysis, as, empirically, neutral trials were not always actually more ambiguous than LF or SN trials for each participant. This is typical for studies using individually tailored indifference 
points (Lin et al., 2018; Kolling et al., 2016; Shenhav, Straccia, Cohen, \& Botvinick, 2014). However, it seems implausible that this phenomenon totally invalidated our results, as the typical conflict effect was present in decision times. Because our null effect does not seem to be an issue of pure power, the added variance by the imprecise tailoring of indifference points does not suffice to explain our results. In addition, we ran a control analysis on a subsample of participants who chose roughly 50:50 in the ambiguous condition, that is, for whom the indifference points estimation was very successful. Even on this subsample, we did not find any effect of ambiguity on theta power (see Appendix B, Figure A5). Another issue may be that our choice ambiguity conditions were not dissimilar enough; a closer look at our data show that we have a similar theta peak to Lin et al. (2018), but this peak was not modulated by our choice ambiguity manipulation. As the theta peak in Lin et al.' work was not perfectly modulated in a parametric manner, it is possible that the differences in choice ambiguity in our task were too small (especially with the added variance from the noisy subjective preference estimates) in order to be tracked by theta.

The second explanation on the theoretical level questions whether value-based conflicts are comparable to inhibitory control conflicts. We postulated that conflict results from any kind of competition on a neural level; therefore, competition because of inhibition and competition because of subjective value similarity should both result in increased conflict. However, recently, it has been suggested that these types of competition may constitute separate processes. This account is based on timecontinuous behavioral measures, such as computer mouse movements of choices. Recording the computer mouse movement when participants indicate their response allows to study the decision-making process in more detail, for example, by measuring how much the mouse movement deviates toward the alternative option, or how much the mouse movement wavers between two options before a final choice is made. Recently, mouse tracking was used to study two different types of conflict in value-based decisions: conflict in the sense of an automatic bias or tendency that needs to be inhibited, and conflict in the sense of an ongoing deliberation process (Cheng \& González-Vallejo, 2018). Superficially, the two conflicts appear comparable, as they are expressed in longer response times. However, in mouse movements, these two types of conflict are expressed in different characteristics. First, the inhibition account translates into an activation of the alternative response/option that competes with the correct/preferable option and which is reflected in a deflection of movement in the direction of the alternative (wrong) response, as is usually found in inhibitory control tasks (Scherbaum et al., 2016; Scherbaum, Dshemuchadse, Fischer, \& Goschke, 2010), and has also be observed in value-based decision tasks (Cheng \& González-Vallejo, 2018; Scherbaum et al.,
2012, 2016; Dshemuchadse, Scherbaum, \& Goschke, 2013; Koop \& Johnson, 2013). The second type of conflict has only been identified for value-based decisions and seems to reflect the ongoing co-activation of relatively similar options - an ongoing process of deliberationwhich is indicated by the amount of wavering in the mouse movement (Cheng \& González-Vallejo, 2018). Our choice ambiguity manipulation seems to match this ongoing deliberation process and might therefore reflect something different to the inhibitory conflict, which is better matched by the repetition bias and the conflict by choosing the delayed (in our case, the LF) over the sooner (in our case, SN) option (Cheng \& González-Vallejo, 2018). In this interpretation, theta oscillations may reflect the first type of conflict, competing activations, and inhibition, whereas the second type, ongoing deliberation, may not be reflected in theta oscillations. Taken together, objective conflict in inhibitory control tasks might not be the same as subjective conflict, similarly to the distinctness of cognitive control and self-control (Scherbaum, Frisch, Holfert, O'Hora, \& Dshemuchadse, 2018).

Next, our task also allowed us to analyze the role of choice repetition effects. We previously demonstrated that people have a tendency to stick to their initial choice (e.g., a soon but small option compared to a late but large option) even when that option gets increasingly less valuable (e.g., the soon option's value decreases from trial to trial; Schoemann \& Scherbaum, 2020; Senftleben et al., 2019; Scherbaum et al., 2016). How does this situation produce conflict? It has been assumed that choice repetition reflects the influence of residual activity from the previous trial (Senftleben et al., 2019; Bonaiuto, de Berker, \& Bestmann, 2016; Rustichini \& PadoaSchioppa, 2015; Gao, Wong-Lin, Holmes, Simen, \& Cohen, 2009), and this residual activation of one option's representation competes with the new incoming activation for the current options' values (we provide a formalized derivation of this hypothesis in Appendix A). On the behavioral level, we found this choice repetition effect on a trial-by-trial basis. This effect was present in all trial types, not just in ambiguous trials. This means that, even if one option was more valuable in the previous trial but was clearly less valuable in the following trial, people still showed a tendency to stick to that option and repeat this choice, even though it was disadvantageous to them. Furthermore, switching to the alternative choice was associated with longer decision times. On the neural level, we found that mid-frontal theta power was increased in the time window of $800-400 \mathrm{msec}$ before the response for trials in which participants switched to the alternative option compared to trials in which participants repeated their previous choice. This indicates that the influence of the previous choice is especially strong relatively early on in the decision-making process (also supported by our regression analysis in Appendix B, Figure A6), as it would be expected for residual activation from the previous trial (Scherbaum et al., 2013). Taking together, for choice 
repetition as potential source of conflict in value-based decision-making, we found evidence on the behavioral as well as on the neural level.

As a final source of conflict, we investigated the effect of temporal delay by comparing decisions for the SN and the LF options. In our task, spatial distance is directly connected to temporal delay: The further away an option is, the longer it takes to collect it. Because participants only have a limited amount of time to collect as much reward as possible, this means that they have to estimate how long it takes to collect each option. Hence, it is this time cost, rather than the spatial distance itself, that is essential for the decision-making process. However, how does choosing the delayed option lead to conflict? One explanation for this is that temporal information is accumulated at a faster rate than value information, possibly because of the nonlinear perception of time (Vincent \& Stewart, 2020; Scherbaum et al., 2012; Zauberman et al., 2009; we provide a formalized derivation of this hypothesis in Appendix A), but explanations can also be derived from dual systems accounts, which postulate a fast, impulsive system and a slow, deliberative system (McClure, Laibson, Loewenstein, \& Cohen, 2004; but see Monterosso \& Luo, 2010; Kable \& Glimcher, 2007, for the intense debate about dual-systems approaches). Both explanations predict an automatic bias toward the sooner option that needs to be overcome or inhibited in order to choose the delayed option. On a behavioral level, we found longer decision times for the delayed options, as expected. This fits well with research on intertemporal choice-choices between smaller rewards that are available sooner and larger rewards that are available later in time. There, it was shown that choosing the larger, delayed option is associated with increased behavioral conflict and longer decision times (Cheng \& González-Vallejo, 2018; O'Hora et al., 2016; Dshemuchadse et al., 2013; Scherbaum et al., 2013; McClure et al., 2004). We also demonstrated this conflict effect on a neural level: Mid-frontal theta power was increased for the delayed option for the majority of the decision-making process (800-124 msec before the response). Hence, choosing the delayed option seems to be associated with increased conflict, just as predicted.

\section{The Role of Mid-frontal Theta}

Mid-frontal theta power is reliably involved in conflict processing (Cooper et al., 2019; Zavala et al., 2018; Pinner \& Cavanagh, 2017; Cavanagh \& Frank, 2014; Cohen \& Donner, 2013; Nigbur et al., 2011). However, it is still unclear what role mid-frontal theta plays. Currently, there are two (not mutually exclusive) perspectives on conflictrelated theta. First, mid-frontal theta has been suggested to act as a conflict monitor or detector: It tracks conflict and increases when conflict increases. This perspective fits well with the reliable increase of mid-frontal theta in highconflict trials of inhibitory control tasks, such as the Stroop
(Zhao et al., 2015; Hanslmayr et al., 2008) or the Flanker task (Pinner \& Cavanagh, 2017; Nigbur et al., 2011; Cohen et al., 2008). In these tasks, mid-frontal theta is typically correlated with RTs (Cohen \& Donner, 2013), and it is specifically the non-phase-locked component of theta that seems to act as a conflict signal (Cohen, 2016; Cohen \& Donner, 2013). In addition, theta also is associated with conflict in more complex control tasks, such as task switching (Cooper et al., 2019; Frisch, Surrey, Dshemuchadse, Maack, \& Scherbaum, 2019). On a neuroanatomical level, mid-frontal theta is thought to originate in the dACC (Töllner et al., 2017; Womelsdorf et al., 2010; Cohen et al., 2008), which is discussed as one of the key structures for conflict monitoring (Shenhav, Botvinick, \& Cohen, 2013; Botvinick, 2007; Botvinick et al., 2004). Based on these findings, it has been suggested that theta bursts serve as a conflict signal, which serves to coordinate brain networks for top-down (inhibitory) control (Verguts, 2017; Cavanagh \& Frank, 2014; Cohen \& Donner, 2013).

The second perspective on conflict-related theta goes further than that and postulates that theta does not merely reflect conflict, but serves as a general alertness or alarm signal (Cavanagh \& Frank, 2014). This perspective is based on research showing that mid-frontal theta also increases under uncertainty or unexpectedness (Lin et al., 2018; Cavanagh, Figueroa, et al., 2012). For example, in Lin et al.' intertemporal choice study (2018), they included "no-brainer" trials (e.g., the choice between $15 €$ now or $15 €$ in 2 weeks), where the decision conflict itself should be very low. Mid-frontal theta power was significantly higher in these no-brainer trials than in real, low-conflict trials, and was similarly high as in highconflict trials. Because no-brainer trials were very rare, this likely reflects a surprise signal. Hence, mid-frontal theta may serve as a signal for additional attention or resources, not just for conflict-related control.

How do our results relate to this discussion? Previous studies investigating conflict-related theta mostly focused on inhibitory control tasks (but see Lin et al., 2018). We extend this research by showing that mid-frontal theta power is sensitive to conflict in a complex value-based decision task. Interestingly, in our task, theta reflected two types of conflicts that can also be interpreted to involve inhibition. First, mid-frontal theta was increased for choice switches as compared to choice repetitions. As humans have an automatic bias to repeat previous choices because of residual activity (Senftleben et al., 2019; Gao et al., 2009; Soetens, Boer, \& Hueting, 1985), switching to the alternative option requires overcomingor inhibiting - this automatic repetition bias. Second, midfrontal theta was increased for delayed as compared to sooner available choices. Research on intertemporal choice has revealed that people have a tendency to prefer the sooner available option (Cheng \& González-Vallejo, 2018; McClure et al., 2004). Hence, in order to choose the delayed option, it is necessary to inhibit this initial bias toward the sooner available option (Scherbaum, Frisch, \& 
Dshemuchadse, 2018). Taken together, our results suggest that mid-frontal theta reflects not only response inhibition but also inhibition of abstract decision biases in a complex value-based decision scenario. Finally, we note that we did not find a theta effect of choice ambiguity, the only conflict type that may rather reflect an ongoing deliberation process and not inhibitory processes, as we discussed above. Therefore, our null effect raises the question if mid-frontal theta truly picks up on deliberation between similar choice options, as was suggested by Lin et al. (Lin et al., 2018), or if this aspect of the decision-making process does not necessarily involve theta. This also highlights how theta may serve as a tool to dissociate different components of complex decision-making processes. Future research should investigate the role of theta in a wider variety of conflict scenarios and paradigms in order to shed light on this issue.

\section{Limitations}

While our experimental design and our paradigm allowed us to investigate different types of conflict within the same value-based decision task, they also come with some limitations that should be addressed. First, mean trial numbers were not always equal within each comparison. In particular, trial counts differed in the comparison between the ambiguous ( $M=66.79$ trials) and unambiguous condition $(M=$ 102.20 trials) because of the fully balanced nature of our target trial manipulation (equal numbers of ambiguous, SN and LF trials; SN and LF were combined for the unambiguous condition; see Table A3 in Appendix B). This should be balanced better in the future by only including half the number of SN and LF target trials.

Second, because of the spatial nature of our decision game, it is possible that eye movements differed systematically between choices of the closer SN option and the far LF option. This could potentially introduce confounds into our EEG data. Because we did not record eye movements, we cannot fully exclude this possibility. However, this should not be an issue for the ambiguity and repetition effects, as SN and LF choices and therefore distances of the reward options were equally distributed for these conditions. In addition, the nature of our task required participants to look at both the SN and the LF option in order to make their first click toward one of these options. Because we only analyze this predecision time from stimulus onset until this first click, we think that even for the SN versus LF comparison, eye movements could be fairly comparable. We performed a control analysis to address this issue (see Appendix B). Specifically, we reasoned that any systematic differences in eye movements would be driven by the distances from the reward options to the avatar (as distances were shorter for LF target choices and longer for SN target choices). Hence, we ran a multiple regression with distance and trial type (SN vs. LF) as regressors on averaged theta power. The analysis did not reveal any effect of distance, but confirmed our finding of increased theta power for LF as compared to SN choices (see Figure A7). This strengthens our case that these effects reflect true cognitive processing and are not merely confound effects of eye movement. Still, this needs to be addressed in future work either by recording eye movements, or by implementing the subjective value manipulation through changing the reward magnitude instead of the reward distance (see Schoemann \& Scherbaum, 2020), so that reward distances can be fully balanced between conditions.

\section{Conclusion}

We investigated conflict in a value-based decision task. We found evidence for a choice repetition bias and for an effect of temporal delay: Behavioral and neural markers of conflict were increased for choice switches (as compared to repetitions) and for sooner available (as compared to later available) choices. We found a behavioral effect of choice ambiguity, which was not reflected in mid-frontal theta power. Taken together, our results suggest that midfrontal theta is sensitive to some, but not all, sources of conflict in value-based decision-making.

\section{APPENDIX A: NEURAL NETWORK SIMULATION OF CONFLICT}

We simulated our hypotheses in a minimal neural network model with attractor dynamics that we already successfully applied to value-based choice in previous studies (Senftleben, Schoemann, Rudolf, \& Scherbaum, 2021; Schoemann \& Scherbaum, 2020; Senftleben et al., 2019; Scherbaum et al., 2016). The model simulates the choice between the SN and LF option as described in the main text. Here, we manipulated model parameters to simulate the three sources of conflict introduced in the main text: choice switches versus repetitions, ambiguous versus unambiguous choices, and sooner versus delayed choices.

We expected choice switches to induce conflict because the residual activity from the previous choice should bias the model toward that choice option, hence leading to more competition between the two choice options in a switch versus in a repetition choice. We expected this effect to emerge naturally from the model, as we showed in our previous simulations (Senftleben et al., 2019, 2021; Schoemann \& Scherbaum, 2020; Scherbaum et al., 2016). We expected ambiguous choices to induce conflict because highly similar input values lead to increased competition between the decision units. We implemented this by manipulating the subjective values for each option, so that the subjective values were either similar (ambiguous) or one subjective value was substantially higher than the other (unambiguous). Finally, we expected temporally delayed choices (LF choices) to lead to more conflict because of differences in the rate of value accumulation. This is based on intertemporal choice 
research showing that humans perceive time in a nonlinear way (Kim \& Zauberman, 2009; Zauberman et al., 2009), which leads to a faster accumulation of temporal information as compared to reward value information (Scherbaum et al., 2012). Because the reduced model we used here did not model the separate accumulation of each attribute (time and reward value), we implemented this effect by manipulating the speed of subjective value accumulation for each unit. The simulation of this effect in the full model is described elsewhere (Scherbaum et al., 2012).

\section{Model Architecture}

Here, we will briefly review the model architecture and its dynamics (for an in-depth description, please see Scherbaum et al., 2016). The model is a connectionist model comparable to the leaky, competing accumulator model (Usher \& McClelland, 2001). The model consists of two units that represent the two choice options (SN and LF). Each unit samples evidence for the respective choice option in the form of the subjective value of that choice option. Through the biophysical properties of lateral inhibition, self-excitation, and nonlinearity, a winnertakes-all dynamic develops that ends with the choice of one option. The model dynamics can be described by two coupled differential equations:

$$
\begin{aligned}
& \tau \dot{u}_{S N}=-u_{S N}+b+w_{r} \cdot \sigma\left(u_{S N}\right)+w_{i} \cdot \sigma\left(u_{L F}\right)+I_{S N} \\
& \tau \dot{u}_{L F}=-u_{L F}+b+w_{r} \cdot \sigma\left(u_{L F}\right)+w_{i} \cdot \sigma\left(u_{S N}\right)+I_{L F}
\end{aligned}
$$

Here, $\tau$ is the time scale (defining the step size of the Euler solution), $b$ is the resting level, $w_{i}$ is the (inhibitory) coupling strength of the two equations, $w_{r}$ is the recurrent feedback, $I_{S N}$ and $I_{L F}$ are the input representing the attractiveness of the two options, and $\sigma$ represents the sigmoidal activation function: $\sigma(u)=\frac{1}{e^{-\beta(u-a)}}$. The model dynamics unfold over time until the activation of one of the units exceeds a threshold, which marks the response. After the response, the inputs for each unit are switched off in order to mimic the ITI (length determined by $t_{i t i}$ ). After the ITI has passed, the inputs for the next trials are switched on and the decision process begins anew. Crucially, we did not reset the model to its initial state, but rather let the model dynamics carry on during the ITI, which is essential for capturing choice repetition effects.

\section{Simulation Methods}

Table A1 gives an overview over parameter values, which are identical to our previous simulations (Senftleben et al., 2019, 2021; except for a stronger noise, which was $N(0,0.1)$ in the original simulation). For the choice ambiguity effect, we manipulated the subjective values of each choice option to create SN, LF, and neutral trials. In $\mathrm{SN}$ trials, the $\mathrm{SN}$ value was higher than the LF value (and
Table A1. Model Parameter Values Used for Simulation

\begin{tabular}{ll}
\hline Parameter & \multicolumn{1}{c}{ Values } \\
\hline$\tau_{\mathrm{LF}}$ & 10 \\
$\tau_{\mathrm{SN}}$ & 9.5 vs. 10 \\
$b$ & -5 \\
$w_{i}$ & -7 \\
$w_{r}$ & 6 \\
$a$ & 0 \\
$\beta$ & $N(1,0.2)$ \\
$\varepsilon^{\mathrm{a}}$ & $N(0,0.2)$ \\
$c$ & -0.12 vs. 0 vs. 0.12 \\
$I$ & 6 \\
$t_{\text {iti }}$ & 55 \\
threshold & 0.85 \\
\hline
\end{tabular}

${ }^{a}$ Indicates parameter values that are not identical to Senftleben et al. (2019, 2021)

vice versa), whereas in neutral trials, both input values were identical. Inputs were defined as: $I_{S N}=I+c$ and $I_{L F}=I-c$, with $I$ being a constant and $c$ determining the subjective value difference $(c>0$ : unambiguous $\mathrm{SN}$ trial, $c<0$ : unambiguous LF trial; $c=0$ : ambiguous/neutral trial). For the temporal delay effect, we systematically varied the time step parameter $\tau$ : In the symmetric condition, $\tau$ was set to 10 for both decision units. In the asymmetric condition, $\tau$ was instead set to 9.5 for the SN option in order to simulate a faster value accumulation for the SN unit.

Based on these manipulations, we created a task design that is comparable to our empirical task design. We built mini-blocks of three trials (two bias trials, one target trial). In each mini-block, bias trials were either $\mathrm{SN}$ or LF, and target trials were either SN, LF, or neutral. The task design allowed us to look at each conflict effect separately. As in the empirical analysis, we compared neutral target trials after SN bias trials and after LF bias trials for the choice repetition effect. For the ambiguity, we compared unambiguous target trials ( $\mathrm{SN}$ and $\mathrm{LF}$ ) with ambiguous target trials (neutral targets). For the temporal delay, we compared SN and LF targets under the asymmetric value accumulation condition.

In order to test our hypotheses, we simulated 20 participants (with interindividual variations in the gain parameter of the sigmoidal activation function $(\beta)$ and time-varying background noise). For each participant, we ran the simulation under each value accumulation condition (symmetric, asymmetric). Within each simulation, the model went through 20 repetitions of the six mini-block scenarios (2 [bias: $\mathrm{SN}$ or $\mathrm{LF}$ ] $\times 3$ [target: $\mathrm{SN}, \mathrm{LF}$, neutral]). The order of the mini-blocks was randomized. As outcomes, we measured the choice (SN or $\mathrm{LF}$ ), the response time (measured in time steps between 
stimulus onset and response), and conflict. In the context of neural network models, conflict can be understood as the amount of competition between the two decision units. If both units have similar levels of activity (e.g., because both decision options are of equal value), competition (and therefore conflict) is high, whereas if one unit has a high level of activity and the other unit has a low level of activity, competition (and therefore conflict) is low. Mathematically, this is usually described as the integrated product of both unit's activity (Gao et al., 2009):

$$
\int \delta\left(u_{1}(t)\right) \delta\left(u_{2}(t)\right) d t
$$

where $\delta$ is the nonlinear activation function, and $u_{1}$ and $u_{2}$ are the values of the decision units at time point $t$. This results in a conflict value for each trial. In addition, we also calculated the maximum conflict value in each trial as a second conflict measure that was not dependent on the trial length. All model simulations were carried out in MATLAB R2017b on a Windows 10 computer, and additional statistical analyses were carried out in JASP Version 0.11.1. The model code and simulated data are openly available at https://osf.io/bphx9/.

\section{Simulation Results}

Figure A1 illustrates the results of the simulation. First, we analyzed choice percentages in the symmetric value accumulation condition (see Figure A1A). We ran a repeatedmeasures ANOVA with the factors bias (SN/LF) and target (SN/LF/neutral) on the LF choice percentages in target trials. As expected, we found a significant main effect for target, $F(2,38)=712.05, p<.001, \eta^{2}=.81$, indicating that the choice ambiguity drove decision-making so that LF was most often chosen in LF trials, SN most often in SN trials, and both choices were roughly equally likely in neutral trials. We also found a significant effect of bias, $F(1$, 19) $=61.64, p<.001, \eta^{2}=.09$, which indicates the choice repetition effect (more LF choices after LF-biased trials as compared to SN-biased trials). The interaction Bias $\times$ Target was also significant, $F(2,38)=5.57, p=.008, \eta^{2}=$ .01 , indicating greater choice repetition for neutral target trials.

Next, we analyzed response times and conflict measures in detail. For the residual activity hypothesis, we compared response times and conflict measures in neutral target choice repetitions versus switches (in the symmetric value accumulation condition). As expected, response times and both conflict measures were significantly smaller in choice repetitions than in switches, all $p s<.001$ (see Figure A1B). For the ambiguity hypothesis, we compared ambiguous (i.e., neutral) target trials with unambiguous (i.e., SN or LF) target trials (in the symmetric value accumulation condition). As expected, response times and both conflict measures were significantly smaller in unambiguous trials, all $p \mathrm{~s}<.001$ (see Figure A1C). Finally, we compared the symmetric and the asymmetric value accumulation condition (see Figure A2). Here, we compared response times and conflict in SN and LF target trials. As expected, SN target choices were significantly faster and lower in conflict, all $p s<.001$ (see Figure A2A-C). In addition, we repeated the choice percentage analysis from above in order to test

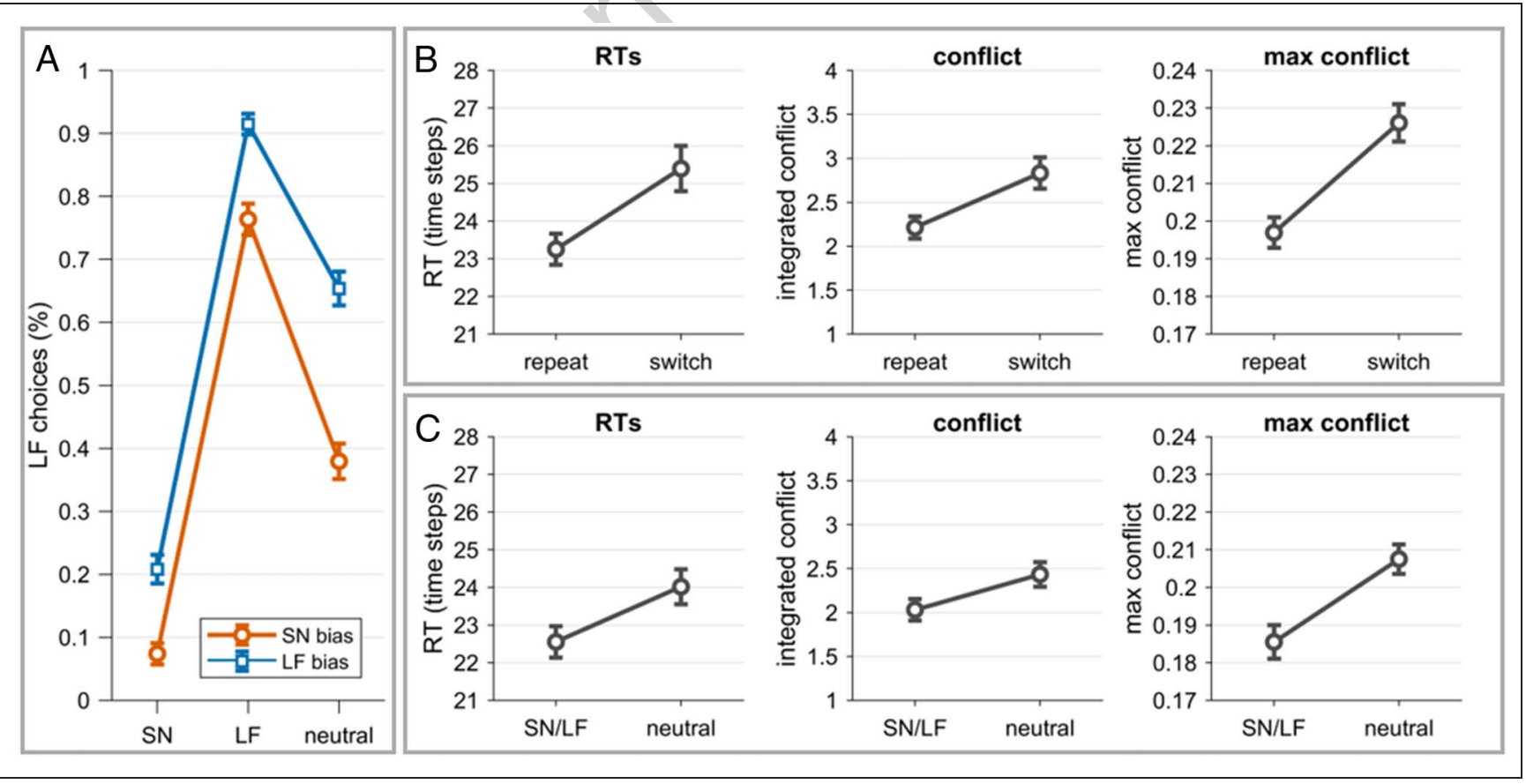

Figure A1. Results of the model simulation (symmetric value accumulation condition). (A) LF choice percentages for SN, LF, and neutral targets, separately for previous SN and LF bias. (B) Choice repetition/residual activity effects. Response times (RTs), integrated conflict, and maximal conflict measures for choice repetitions and choice switches in neutral target trials. (C) Choice ambiguity effects. RTs, integrated conflict, and maximal conflict measures for unambiguous ( $\mathrm{SN} / \mathrm{LF}$ ) and neutral target trials. All error bars represent standard errors. 
Figure A2. Asymmetric value accumulation condition. (A) LF choice percentages in $\mathrm{SN}$, $\mathrm{LF}$, and neutral target trials, separately for $\mathrm{SN}$ and LF bias trials. (B) Response times (RT) for $\mathrm{SN}$ and LF target trials. All error bars represent standard errors.

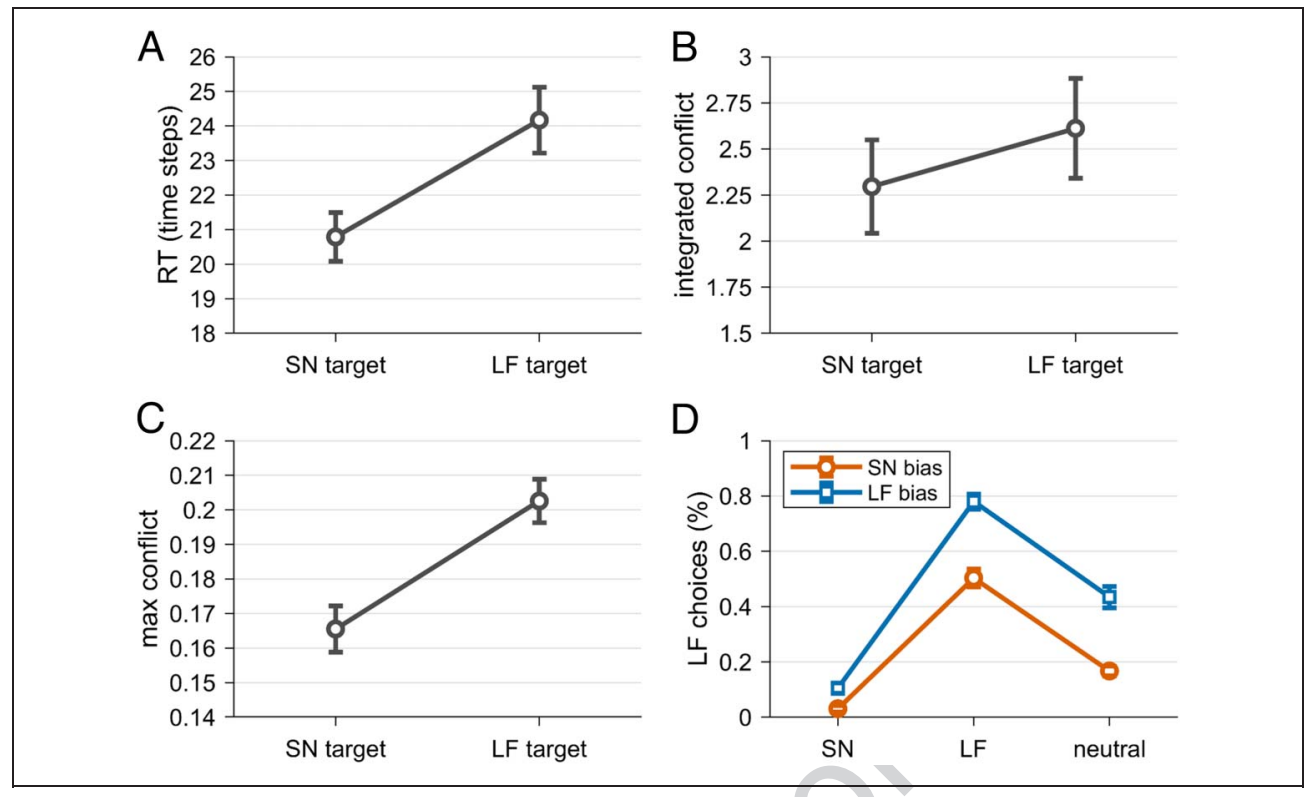

if the asymmetric value accumulation would interfere with the choice repetition effect (see Figure A2D). The repeated-measures ANOVA revealed the expected significant main effects of bias, $F(1,19)=68.32, p<.001, \eta^{2}=$ .08 , and target, $F(2,38)=409.25, p<.001, \eta^{2}=.79$, as well as the interaction Bias $\times$ Target, $F(2,38)=14.06$, $p<.001, \eta^{2}=.02$. Hence, the model produces choice repetition effects even under asymmetric value accumulation.

\section{Conclusions}

In summary, our simulation demonstrated that our hypotheses regarding conflict in value-based decisions are directly predicted by a minimal neural network model with attractor dynamics. The model produced choice repetition, choice ambiguity, and temporal delay effects in response times and in conflict, as measured as the product of activity. Although we did not model specific anatomical brain regions, our model captures essential properties and dynamics of neuronal populations, namely, lateral inhibition, self-excitation, and nonlinearity. As conflict signals have been associated with increased mid-frontal theta power and increased activity in the $\mathrm{dACC}$ and $\mathrm{mPFC}$, we expect that mid-frontal theta power should be increased in all conditions where we found increased conflict in our neural model: for choice switches versus repetitions, for ambiguous versus unambiguous choices, and for delayed versus sooner available choices.

\section{APPENDIX B: EMPIRICAL RESULTS}

\section{Estimation of Indifference Points}

Indifference points describe the value difference of the SN option and the LF option where participants are indifferent, that is, the probability of choosing the SN option over the LF option is $50 \%$. Indifference points were estimated separately for each distance. For the estimation of the indifference points at a certain distance, we determined the point of inflection of a logistic function that was fitted to each participant's choice data (SN option vs. LF option) as a function of increasing value differences. This was carried out using the function logitfit from the StixBox mathematical toolbox by Anders Holtsberg (https://www.maths.lth.se/matstat/stixbox/). The fit was based on the model $\log [p /(1-p)]=b 0+$ $X * b 1$, where $p$ is the probability that the choice is 1 (SN option) and not 0 (LF option), $X$ represents value differences, and $b 0$ and $b 1$ represent the point estimates for the logistic function. In the experiment, indifference points were calculated after the measurement block in order to manipulate choices in the subsequent experimental block. For that calculation, we discarded the last 10 trials of the measurement block because these trials might reflect time pressure more than they might reflect actual subjective values, and this was shown to improve the subsequent manipulation in our previous studies (Senftleben et al., 2021).

\section{Measurement Block - Discounting}

Based on the indifference points estimated from the measurement block, the discounting parameter $k$ can be obtained. This discounting parameter is typically used to describe discounting in intertemporal choice. It is obtained by fitting a hyperbolic model to the indifference points of each participant. This hyperbolic model assumes that participants prefer the LF option less and less the larger the delay to the LF option becomes (in our paradigm: the larger the distance to the LF option becomes). The parameter $k$ represents how steep this function is: A higher $k$ corresponds to a higher devaluation of the delayed reward (i.e., stronger preference for $\mathrm{SN}$ choices, especially when the LF option is far away). 
Table A2. Behavioral Results

\begin{tabular}{lcc}
\hline & $\begin{array}{c}\text { Measurement } \\
\text { Block }\end{array}$ & $\begin{array}{c}\text { Experimental } \\
\text { Block }\end{array}$ \\
\hline $\begin{array}{c}\text { Mean amount of } \\
\text { trials }(S D)\end{array}$ & $172.60(31.97)$ & $615.69(99.27)$ \\
$\begin{array}{l}\text { Mean amount of } \\
\text { SN choices }(S D)\end{array}$ & $106.54(42.18)$ & $314.21(129.85)$ \\
$\begin{array}{l}\text { Mean amount of } \\
\text { LF choices }(S D)\end{array}$ & $66.06(17.08)$ & $301.48(80.06)$ \\
Mean $k$ & $.23(.18)$ & N/A \\
\hline
\end{tabular}

Table A2 gives an overview of the mean $k$ values as well as other details on participants' behavior in the measurement block (as well as the experimental block). Taken together, this indicates that 1) participants found the LF option less valuable the farther away it was (comparable to how people find monetary rewards less valuable the longer they have to wait for them) and that 2) this devaluation or discounting of the LF option was a stable preference across time.

\section{Experimental Block: Behavioral Data}

Figure A3 shows the repetition effect for each participant as indicated by the repetition index (percentage of LF choices after LF bias trials - percentage of LF choices after SN bias trials) in neutral target trials. In both sessions, the vast majority of participants (around 80\%) showed a choice repetition bias as indicated by a positive repetition index.

Figure A4 shows LF choice percentages for each target type: SN, neutral, and LF. These target trials were created based on each participant's individual choice preference, as indicated by indifference points. In SN trials, the subjective value of the SN option was larger than the indifference point; in LF trials, the subjective value of the SN option was below the indifference point; and in neutral trials, the subjective value of the SN option was directly at the indifference point (i.e., equal subjective values of the SN and LF option). Therefore, participants should choose the SN option in SN trials, the LF option in LF trials, and should have equal SN and LF choice percentages in neutral target trials. Although this manipulation was successful on average (see Figure 3A), there were substantial differences on a single subject level as is illustrated by Figure A4. For example, Participant 6 only chose the LF option in roughly $50 \%$ of LF trials, indicating that LF trial choices were right at their empirical indifference point. Hence, for Participant 6, the LF trial was more ambiguous than the neutral trial because of inaccurate theoretical indifference point estimates. There seemed to be no general over- or underestimation of indifference points, as evidenced by the mean choice percentages (see Behavioral Results section and Figure 3A); however, this means that our categories of ambiguous (neutral) and unambiguous (LF/SN) were not accurate for every participant.

\section{EEG Control Analyses: Ambiguity Effect}

One potential explanation for our null effect of ambiguity on theta power might be the noise within the indifference points estimation, as illustrated in Figure A4. Hence, we ran a control analysis on the participants whose choice behavior in ambiguous trials was roughly 50:50, as predicted if the indifference points were estimated precisely. We included all participants whose percentage of LF choices was within $10 \%$ of the expected $50 \%$. This yielded 24 participants within the behavioral sample and 23 participants within the EEG sample. We reasoned that these participants should uniformly experience high conflict during ambiguous choices and low conflict during unambiguous choices. We reran both the behavioral and the EEG analysis on this subsample. On a behavioral level, we found the expected effect of slower decision times for ambiguous
Figure A3. Repetition index for single participants. The repetition index is defined as the percentage of LF choices after LF bias trials minus the percentage of LF choices after $\mathrm{SN}$ bias trials (a positive value indicates choice repetition).

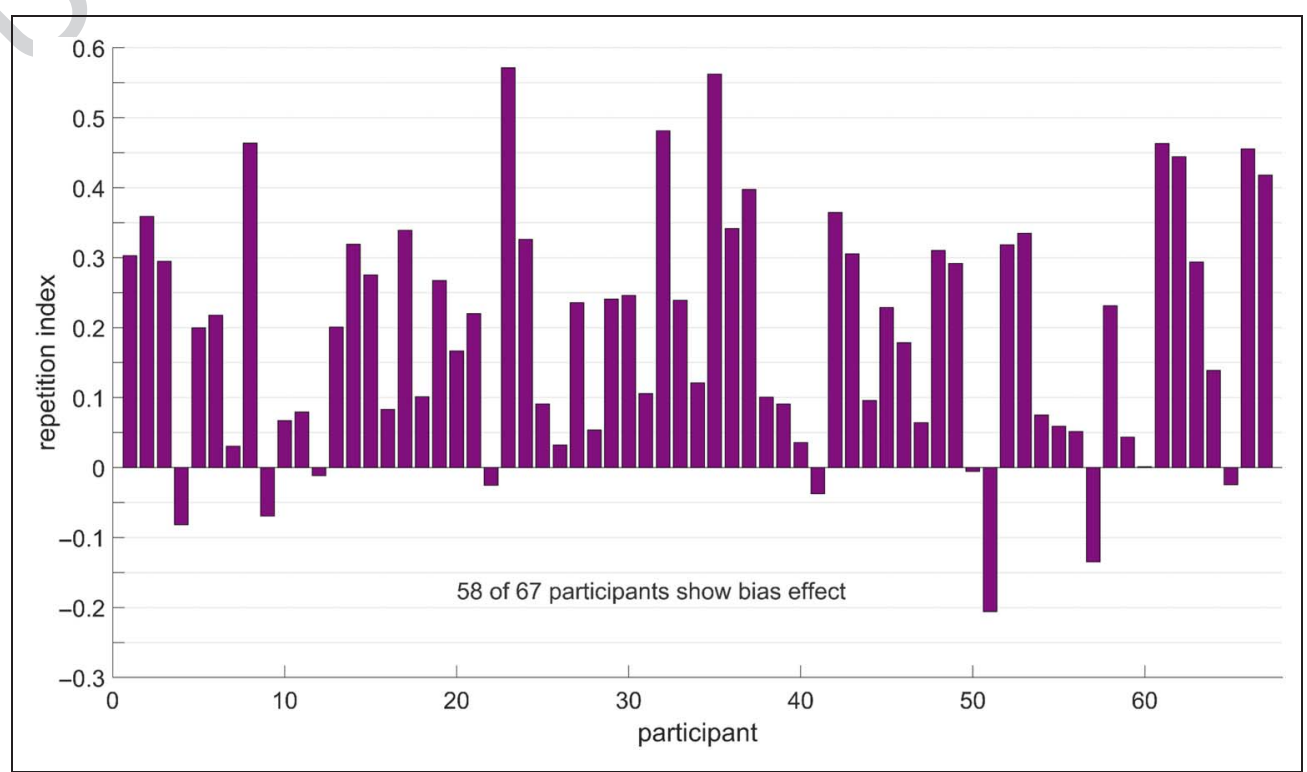




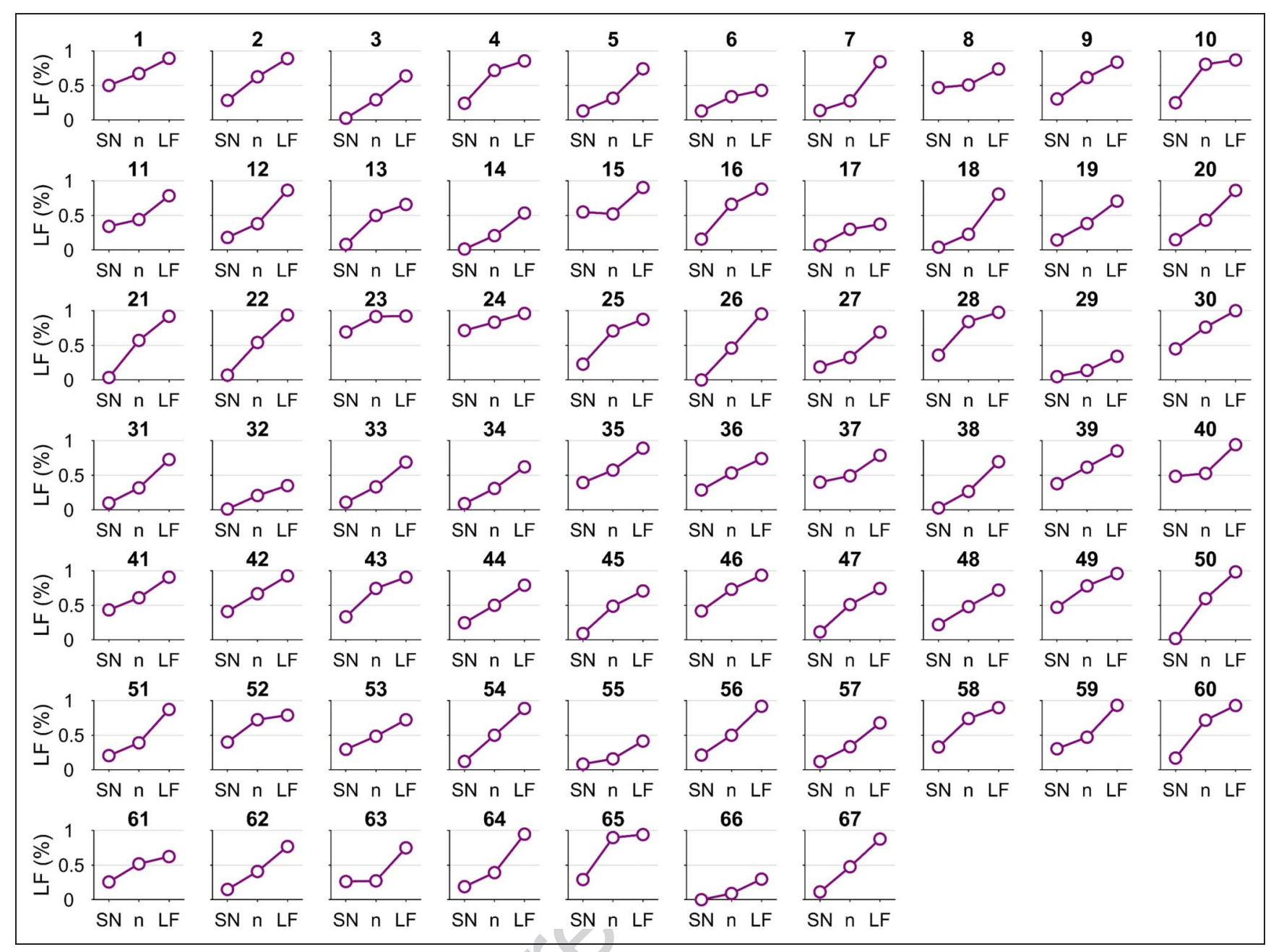

Figure A4. LF choice percentages in SN, neutral (n), and LF target trials for each participant $(n=67)$.

Table A3. Notes to Trial Numbers and Inclusion Criteria for Each Comparison

\begin{tabular}{|c|c|c|c|c|}
\hline Comparison & Condition & $\begin{array}{l}\text { Mean Trial Count } \\
\text { (SD) Behavioral }\end{array}$ & $\begin{array}{c}\text { Mean Trial } \\
\text { Count (SD) EEG }\end{array}$ & Trial Inclusion Criteria \\
\hline \multirow[t]{2}{*}{ Choice ambiguity } & Ambiguous & $66.67(11.16)$ & $66.79(11.33)$ & Ambiguous target trials \\
\hline & Unambiguous & $104.96(17.91)$ & $102.20(19.20)$ & $\begin{array}{l}\text { Unambiguous target trials (SN and LF) with } \\
\text { successful target manipulation (SN choice in } \\
\text { SN target trial, LF choice in LF target trial) }\end{array}$ \\
\hline \multirow[t]{2}{*}{ Choice repetition } & Repetition & $32.87(7.25)$ & $32.88(7.21)$ & $\begin{array}{l}\text { Ambiguous target trials where choice }(\mathrm{t})= \\
\text { choice }(t-1) \text {, and successful bias in } \\
\text { choice }(t-1 \text {; SN choice in SN bias trial, } \\
\text { LF choice in LF bias trial) }\end{array}$ \\
\hline & Switch & $20.55(7.21)$ & $20.77(7.33)$ & $\begin{array}{l}\text { Ambiguous target trials where choice }(\mathrm{t}) \sim= \\
\text { choice }(t-1) \text {, and successful bias in } \\
\text { choice }(t-1 \text {; SN choice in SN bias trial, } \\
\text { LF choice in LF bias trial) }\end{array}$ \\
\hline \multirow[t]{2}{*}{ Temporal delay } & SN & $52.51(16.20)$ & $51.18(16.24)$ & Successful SN target trials (participants chose SN) \\
\hline & $\mathrm{LF}$ & $52.45(11.15)$ & $51.02(12.09)$ & Successful LF target trials (participants chose LF) \\
\hline
\end{tabular}

These trial numbers are based on the behavioral analyses; trial numbers differ slightly for EEG analysis because of the difference in sample $(n=66$ instead of $n=67$ ) and trials omitted because of EEG artifacts. Target trials were the third trial of each mini-block. $S D=$ standard deviation. 

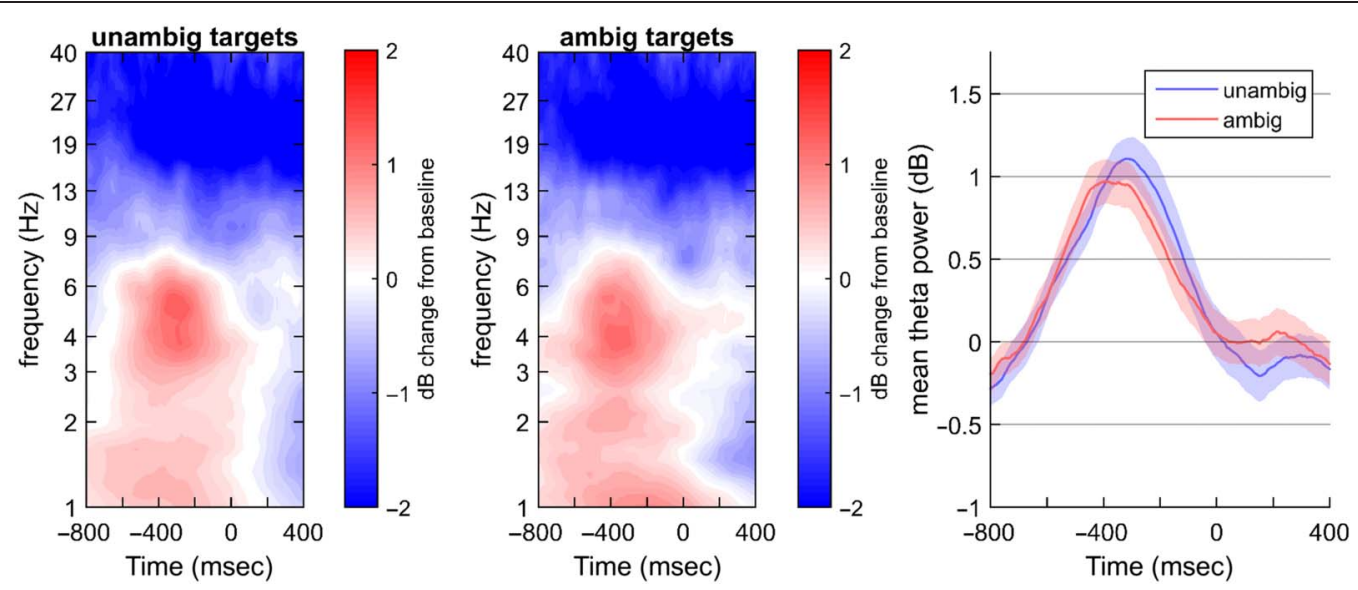

Figure A5. Time-frequency analysis results of choice repetitions and choice switches in ambiguous target trials, for a subsample with the strongest ambiguity manipulation success. All plots are response-locked (0 msec corresponds to the first response). (A) and (B) show dB-converted changes in power as compared to baseline ( -400 to $-200 \mathrm{msec}$ before stimulus onset) for ambiguous (ambig) and unambiguous (unambig) target choices. (C) Averaged theta power $(4-7 \mathrm{~Hz})$, error areas represent standard errors.

choices, $t(23)=4.41, p<.001, g=.27$. However, the EEG analysis again did not reveal any significant differences in mean theta power between ambiguous and unambiguous choices (see Figure A5).

\section{EEG Control Analyses: Multiple Linear Regression}

\section{Distinct Time-Frequency Profiles}

In our main analyses, we tested each of our hypothesis with a planned comparison of average mid-frontal theta power between the relevant conditions (ambiguity: ambiguous vs. unambiguous target choices; repetition: repetitions vs. switches in ambiguous target choices; temporal delay: SN vs. LF option). In order to gain more detailed insights into these effects, we also ran a multiple linear regression on lower $(4-5.5 \mathrm{~Hz})$ and upper $(5.5-7 \mathrm{~Hz})$ theta power. We included all target trials with successful trial type manipulation (i.e., SN targets with SN choice, LF targets with LF choice, all ambiguous trials) and with successful trial type manipulation in the directly preceding bias trial (trial $n-$ $1)$. As regressors, we included repetition and trial type. We coded repetition as $0=$ switch and $1=$ repetition. We coded trial type using Helmert coding, which yielded two regressors (Regressor 1: ambiguous vs. unambiguous [mean of SN and LF]; Regressor 2: SN vs. LF). We ran a multiple linear regression for each time-step on a single-trial basis, for each participant and each theta band. We employed temporal smoothing (Gaussian with width of 10 time-steps; Scherbaum \& Dshemuchadse, 2020). We averaged the resulting beta weights across participants and calculated running $t$ tests against 0 to determine time windows of significance (uncorrected).

The results are presented in Figure A6, and are largely consistent with the results of our main analysis. Ambiguity had no effect during the decision-making process. Roughly $200 \mathrm{msec}$ after the response, we found an effect of increased theta power (lower band) for ambiguous choices. We did not find this effect in our main analysis, but it would be consistent with a postresponse evaluation or monitoring process (however, we need to stress that this result needs to be interpreted very cautiously). As expected, the repetition effect predicted less theta power for repetitions early in the decision-making process; this pattern was present for both lower and upper theta band, although it only reached statistical significance for lower theta. The brief segment of increased lower theta power for repetitions at around $200 \mathrm{msec}$ after the response is not consistent with our main analysis; however, this segment is relatively brief and may reflect pure noise (as we present uncorrected significance test results). Finally, the temporal delay regressor predicted decreased theta power for SN versus LF choices across both the lower and upper theta band during large parts of the decision-making process. This is consistent with the results of our main analyses. In conclusion, the regression analysis shows that the three conflict effects we studied here have distinct temporal profiles. As only the temporal delay regressor ( $\mathrm{SN}$ vs. LF) reached significance for both lower and upper theta band, this raises the possibility that these effects also peak at distinct theta-frequencies; however, as the general pattern of the temporal profile for the three regressors was qualitatively comparable between lower and upper theta band, we think that this may also simply be an issue of noise. Therefore, our results make a first step in identifying potentially distinct time-frequency profiles for different types of conflict in value-based decisions, but more research is necessary to address this question in detail.

\section{Influence of Distance}

Because of the spatial nature of our paradigm, we cannot exclude the possibility that eye movements differed 


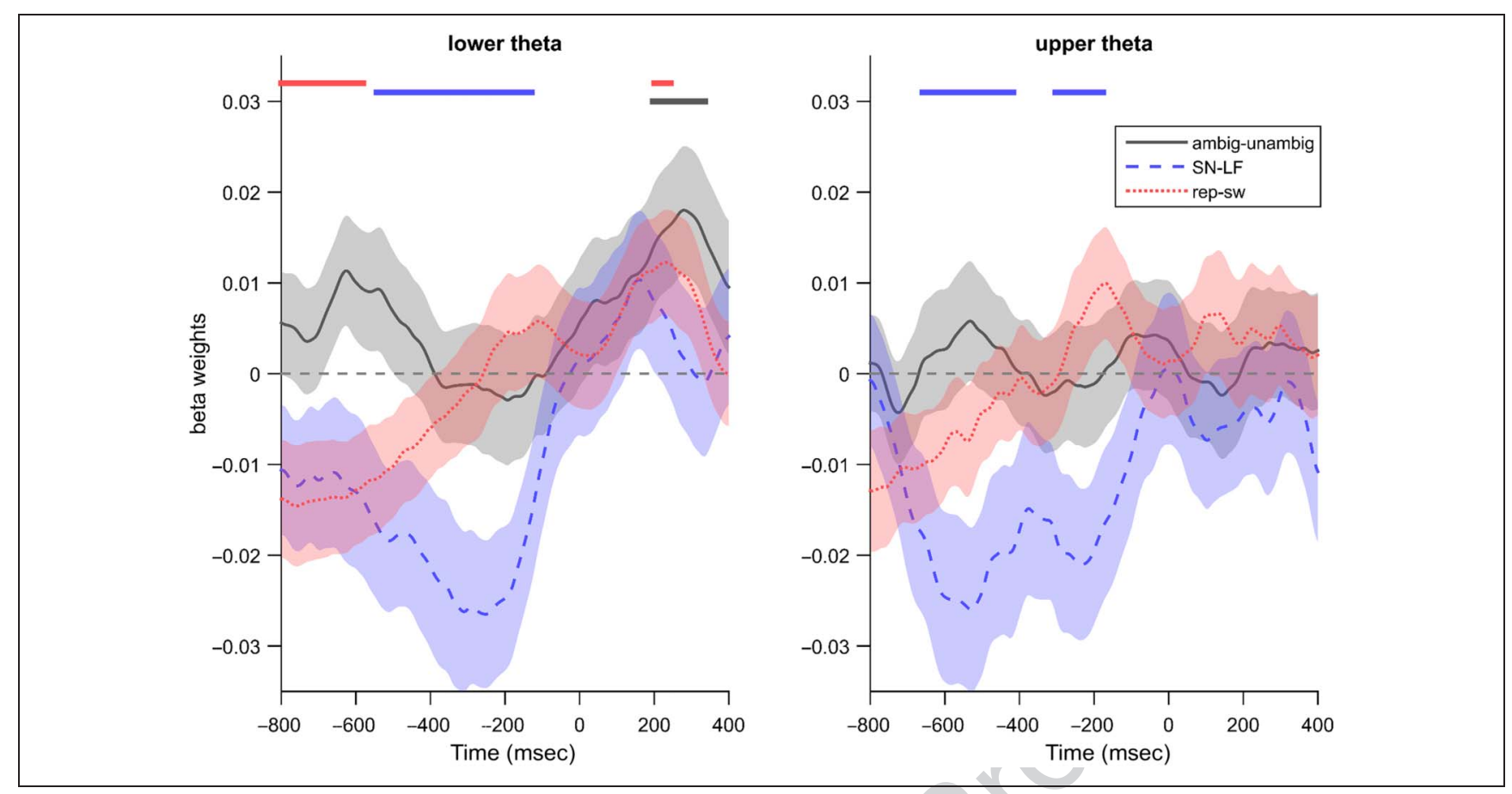

Figure A6. Average beta weights for multiple linear regressions on average lower theta power $(4-5.5 \mathrm{~Hz})$ and average upper theta power $(5.5-7 \mathrm{~Hz})$, time-locked to the response (first click) at $0 \mathrm{msec}$. Beta weights for the regressor of ambiguous (ambig) versus unambiguous (unambig) are represented as solid/gray; SN versus LF is represented as dashed/blue; and repetition (rep) versus switches (sw) are represented as dotted/red. Segments of significance are highlighted by bars in the respective color at the top of each panel. Shaded error areas represent standard errors.

between conditions and, in turn, pose a potential confound for our mid-frontal theta effects. As we discuss in the limitations section of our article, this is less of a problem for comparisons of repetitions versus switches and ambiguous versus unambiguous choices, as the distances from the avatar to the reward options are relatively balanced between these conditions. However, distances are typically shorter for LF target trials and longer for

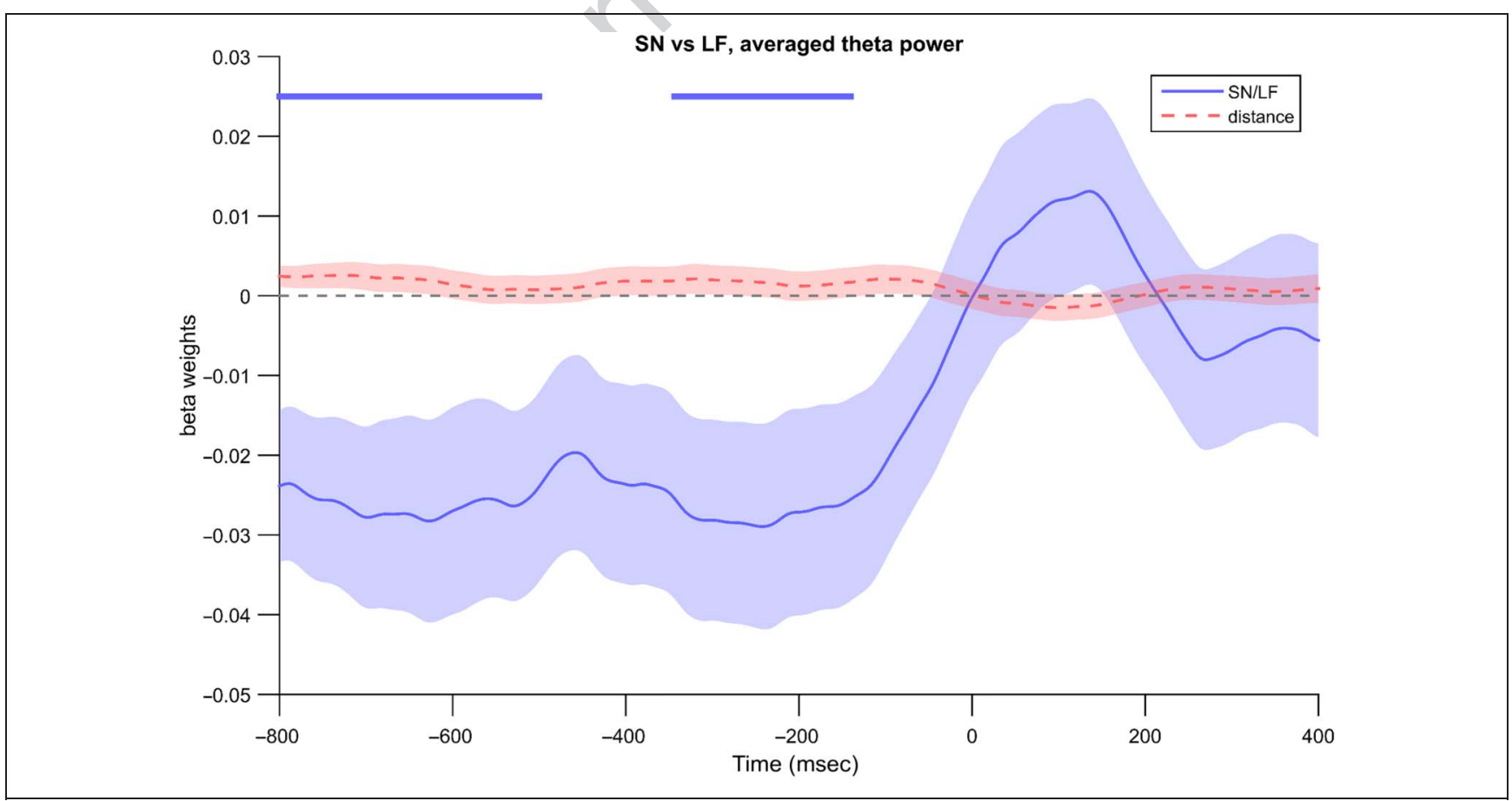

Figure A7. Average beta weights for multiple linear regressions on average theta power, time-locked to the response (first click) at 0 msec. Beta weights for the regressor of trial type are represented as solid/blue; the regressor distance is represented in dashed/red. Segments of significance are highlighted by bars in the respective color at the top of the figure. Shaded error areas represent standard errors. 
SN target trials (because the LF option typically gets more attractive if it is closer; therefore, distances in LF target trials tend to be shorter). Hence, if the distances from the avatar to the reward options lead to distinct eye movements, this could be a serious confound for the $\mathrm{SN}$ versus $\mathrm{LF}$ theta effect. In order to address this issue, we ran multiple linear regression analyses on averaged theta power in SN and LF target choices with the regressors trial type $(\mathrm{SN}=1, \mathrm{LF}=0)$ and distance (112 fields; distance here describes the difference of distance from the LF option to the avatar minus the distance from the SN option to the avatar). We ran this analysis on a single-trial basis for each time-step of each participant. We employed temporal smoothing (Gaussian with width of 10 time-steps), and we checked that all variance inflation factors were below 5 (Scherbaum \& Dshemuchadse, 2020). We averaged resulting beta weights across participants and calculated running $t$ tests against 0 to determine time windows of significance (uncorrected).

The results are presented in Figure A7. The trial type regressor ( $\mathrm{SN}$ vs. LF) reached significance in two time windows, whereas the distance regressor did not reach significance for any time window. Hence, the analysis confirms the effect of reduced mid-frontal theta power for $\mathrm{SN}$ choices as compared to LF choices while not providing any evidence for a confounding effect of distance. This supports that our findings likely reflect cognitive processing and are not merely a result of a confound with eye movements.

\section{Acknowledgments}

This research was supported by the German Research Foundation (DFG; grant SFB 940/2, project A8).

Reprint requests should be sent to Ulrike Senftleben, Department of Psychology, Technische Universität Dresden, 01062 Dresden, Germany, or via e-mail: ulrike.senftleben@tu-dresden.de.

\section{Author Contributions}

Ulrike Senftleben: Conceptualization; Formal analysis; Investigation; Methodology; Project administration; Visualization; Writing-Original draft; Writing-Review \& editing. Stefan Scherbaum: Conceptualization; Funding acquisition; Methodology; Supervision; Writing-Original draft; Writing—Review \& editing.

\section{Funding Information}

Ulrike Senftleben, Deutsche Forschungsgemeinschaft (https://dx.doi.org/10.13039/501100001659), grant number: SFB 940/2 A8.

\section{Diversity in Citation Practices}

A retrospective analysis of the citations in every article published in this journal from 2010 to 2020 has revealed a persistent pattern of gender imbalance: Although the proportions of authorship teams (categorized by estimated gender identification of first author/last author) publishing in the Journal of Cognitive Neuroscience (JoCN) during this period were $\mathrm{M}(\mathrm{an}) / \mathrm{M}=.408, \mathrm{~W}(\mathrm{oman}) / \mathrm{M}=$ $.335, \mathrm{M} / \mathrm{W}=.108$, and $\mathrm{W} / \mathrm{W}=.149$, the comparable proportions for the articles that these authorship teams cited were $\mathrm{M} / \mathrm{M}=.579, \mathrm{~W} / \mathrm{M}=.243, \mathrm{M} / \mathrm{W}=.102$, and $\mathrm{W} / \mathrm{W}=$ .076 (Fulvio et al., JoCN, 33:1, pp. 3-7). Consequently, JoCN encourages all authors to consider gender balance explicitly when selecting which articles to cite and gives them the opportunity to report their article's gender citation balance. The authors of this article report its proportions of citations by gender category to be as follows: $\mathrm{M} / \mathrm{M}=.764, \mathrm{~W} / \mathrm{M}=.073, \mathrm{M} / \mathrm{W}=.127$, and $\mathrm{W} / \mathrm{W}=.036$.

\section{Note}

1. We interpolated in the following ways: If the measured indifference points were monotonically decreasing, we interpolated cubically using the "pchip" method within the function interp1 in MATLAB. If the measured indifference points were not monotonically decreasing, we fit them to an exponential and a hyperbolic model. The model that provided the better fit (as quantified by standard errors) was used to interpolate the indifference points.

\section{REFERENCES}

Bonaiuto, J. J., de Berker, A., \& Bestmann, S. (2016). Response repetition biases in human perceptual decisions are explained by activity decay in competitive attractor models. eLife, 5, e20047. https://doi.org/10.7554/eLife.20047, PubMed: 28005007

Botvinick, M. M. (2007). Conflict monitoring and decision making: Reconciling two perspectives on anterior cingulate function. Cognitive, Affective, \& Behavioral Neuroscience, 7, 356-366. https://doi.org/10.3758/CABN.7.4.356, PubMed: 18189009

Botvinick, M. M., Braver, T. S., Barch, D. M., Carter, C. S., \& Cohen, J. D. (2001). Conflict monitoring and cognitive control. Psychological Review, 108, 624-652. https://doi.org /10.1037/0033-295X.108.3.624, PubMed: 11488380

Botvinick, M. M., Cohen, J. D., \& Carter, C. S. (2004). Conflict monitoring and anterior cingulate cortex: An update. Trends in Cognitive Sciences, 8, 539-546. https://doi.org/10.1016/j .tics.2004.10.003, PubMed: 15556023

Brainard, D. H. (1997). The Psychophysics Toolbox. Spatial Vision, 10, 433-436. https://doi.org/10.1163 /156856897X00357, PubMed: 9176952

Canolty, R. T., Edwards, E., Dalal, S. S., Soltani, M., Nagarajan, S. S., Kirsch, H. E., et al. (2006). High gamma power is phase-locked to theta oscillations in human neocortex. Science, 313, 1626-1628. https://doi.org/10.1126/science.1128115, PubMed: 16973878

Cavanagh, J. F., Figueroa, C. M., Cohen, M. X., \& Frank, M. J. (2012). Frontal theta reflects uncertainty and unexpectedness during exploration and exploitation. Cerebral Cortex, 22, 2575-2586. https://doi.org/10.1093/cercor/bhr332, PubMed: 22120491

Cavanagh, J. F., \& Frank, M. J. (2014). Frontal theta as a mechanism for cognitive control. Trends in Cognitive Sciences, 18, 414-421. https://doi.org/10.1016/j.tics.2014.04 .012, PubMed: 24835663 
Cavanagh, J. F., Zambrano-Vazquez, L., \& Allen, J. J. B. (2012). Theta lingua franca: A common mid-frontal substrate for action monitoring processes. Psychophysiology, 49, 220-238. https://doi.org/10.1111/j.1469-8986.2011.01293.x, PubMed: 22091878

Cheng, J., \& González-Vallejo, C. (2018). Unpacking decision difficulty: Testing action dynamics in intertemporal, gamble, and consumer choices. Acta Psychologica, 190, 199-216. https://doi.org/10.1016/j.actpsy.2018.08.002, PubMed: 30142496

Cohen, J. D., Dunbar, K., \& McClelland, J. L. (1990). On the control of automatic processes: A parallel distributed processing account of the Stroop effect. Psychological Review, 97, 332-361. https://doi.org/10.1037/0033-295X.97.3 .332, PubMed: 2200075

Cohen, M. X. (2016). Midfrontal theta tracks action monitoring over multiple interactive time scales. Neuroimage, 141, 262-272. https://doi.org/10.1016/j.neuroimage.2016.07.054, PubMed: 27475291

Cohen, M. X., \& Cavanagh, J. F. (2011). Single-trial regression elucidates the role of prefrontal theta oscillations in response conflict. Frontiers in Psychology, 2, 30. https://doi.org/10 .3389/fpsyg.2011.00030, PubMed: 21713190

Cohen, M. X., \& Donner, T. H. (2013). Midfrontal conflictrelated theta-band power reflects neural oscillations that predict behavior. Journal of Neurophysiology, 110, 2752-2763. https://doi.org/10.1152/jn.00479.2013, PubMed: 24068756

Cohen, M. X., Ridderinkhof, K. R., Haupt, S., Elger, C. E., \& Fell, J. (2008). Medial frontal cortex and response conflict: Evidence from human intracranial EEG and medial frontal cortex lesion. Brain Research, 1238, 127-142. https://doi.org /10.1016/j.brainres.2008.07.114, PubMed: 18760262

Cooper, P. S., Karayanidis, F., McKewen, M., McLellan-Hall, S., Wong, A. S. W., Skippen, P., et al. (2019). Frontal theta predicts specific cognitive control-induced behavioural changes beyond general reaction time slowing. Neuroimage, 189, 130-140. https://doi.org/10.1016/j.neuroimage.2019.01 .022, PubMed: 30639331

Delorme, A., \& Makeig, S. (2004). EEGLAB: An open source toolbox for analysis of single-trial EEG dynamics including independent component analysis. Journal of Neuroscience Methods, 134, 9-21. https://doi.org/10.1016/j.jneumeth.2003 .10.009, PubMed: 15102499

Dshemuchadse, M., Scherbaum, S., \& Goschke, T. (2013). How decisions emerge: Action dynamics in intertemporal decision making. Journal of Experimental Psychology: General, 142, 93-100. https://doi.org/10.1037/a0028499, PubMed: 22612767

Eriksen, B. A., \& Eriksen, C. W. (1974). Effects of noise letters upon the identification of a target letter in a nonsearch task Perception \& Psychophysics, 16, 143-149. https://doi.org/10 .3758/BF03203267

Frisch, S., Surrey, C., Dshemuchadse, M., Maack, M. C., \& Scherbaum, S. (2019). Frontal midline theta reveals temporal dynamics of target amplification and distracter inhibition during mental set-shifting. PsyArXiv Preprints. https://doi.org /10.31234/osf.io/zt2jb

Gao, J., Wong-Lin, K., Holmes, P., Simen, P., \& Cohen, J. D. (2009). Sequential effects in two-choice reaction time tasks: Decomposition and synthesis of mechanisms. Neural Computation, 21, 2407-2436. https://doi.org/10.1162/neco 2009.09-08-866, PubMed: 19548803

Glöckner, A., Hilbig, B. E., \& Jekel, M. (2014). What is adaptive about adaptive decision making? A parallel constraint satisfaction account. Cognition, 133, 641-666. https://doi.org /10.1016/j.cognition.2014.08.017, PubMed: 25243773

Glöckner, A., \& Pachur, T. (2012). Cognitive models of risky choice: Parameter stability and predictive accuracy of prospect theory. Cognition, 123, 21-32. https://doi.org/10 .1016/j.cognition.2011.12.002, PubMed: 22226615

Hanslmayr, S., Pastötter, B., Bäuml, K.-H., Gruber, S., Wimber, M., \& Klimesch, W. (2008). The electrophysiological dynamics of interference during the Stroop task. Journal of Cognitive Neuroscience, 20, 215-225. https://doi.org/10.1162 /jocn.2008.20020, PubMed: 18275330

JASP Team. (2019). JASP (Version 0.11.1) [Computer software]. Available at: https://jasp-stats.org/.

Jocham, G., Hunt, L. T., Near, J., \& Behrens, T. E. J. (2012). A mechanism for value-guided choice based on the excitationinhibition balance in prefrontal cortex. Nature Neuroscience, 15, 960-961. https://doi.org/10.1038/nn.3140, PubMed: 22706268

Jones, A. D., Cho, R. Y., Nystrom, L. E., Cohen, J. D., \& Braver, T. S. (2002). A computational model of anterior cingulate function in speeded response tasks: Effects of frequency, sequence, and conflict. Cognitive, Affective, \& Behavioral Neuroscience, 2, 300-317. https://doi.org/10.3758/CABN.2.4 300, PubMed: 12641175

Kable, J. W., \& Glimcher, P. W. (2007). The neural correlates of subjective value during intertemporal choice. Nature Neuroscience, 10, 1625-1633. https://doi.org/10.1038/nn2007, PubMed: 17982449

Kim, B. K., \& Zauberman, G. (2009). Perception of anticipatory time in temporal discounting. Journal of Neuroscience, Psychology, and Economics, 2, 91-101. https://doi.org/10 1037/a0017686

Kleiner, M., Brainard, D., Pelli, D., Ingling, A., Murray, R., \& Broussard, C. (2007). What's new in Psychtoolbox-3? A free cross-platform toolkit for psychophysics with MATLAB and GNU/Octave. Perception, 36, 1-16.

Kolling, N., Wittmann, M. K., Behrens, T. E. J., Boorman, E. D., Mars, R. B., \& Rushworth, M. F. S. (2016). Value, search, persistence and model updating in anterior cingulate cortex. Nature Neuroscience, 19, 1280-1285. https://doi.org/10.1038 /nn.4382, PubMed: 27669988

Koop, G. J., \& Johnson, J. G. (2013). The response dynamics of preferential choice. Cognitive Psychology, 67, 151-185. https:// doi.org/10.1016/j.cogpsych.2013.09.001, PubMed: 24128613

Kovacevic, S., Azma, S., Irimia, A., Sherfey, J., Halgren, E., \& Marinkovic, K. (2012). Theta oscillations are sensitive to both early and late conflict processing stages: Effects of alcohol intoxication. PLoS One, 7, e43957. https://doi.org/10.1371 /journal.pone.0043957, PubMed: 22952823

Lin, H., Saunders, B., Hutcherson, C. A., \& Inzlicht, M. (2018). Midfrontal theta and pupil dilation parametrically track subjective conflict (but also surprise) during intertemporal choice. Neuroimage, 172, 838-852. https://doi.org/10.1016/j neuroimage.2017.10.055, PubMed: 29107773

Lisman, J. E., \& Jensen, O. (2013). The theta-gamma neural code. Neuron, 77, 1002-1016. https://doi.org/10.1016/j .neuron.2013.03.007, PubMed: 23522038

Marco-Pallarés, J., Mohammadi, B., Samii, A., \& Münte, T. F. (2010). Brain activations reflect individual discount rates in intertemporal choice. Brain Research, 1320, 123-129. https://doi.org/10.1016/j.brainres.2010.01.025, PubMed: 20083092

McClure, S. M., Laibson, D. I., Loewenstein, G., \& Cohen, J. D. (2004). Separate neural systems value immediate and delayed monetary rewards. Science, 306, 503-507. https://doi.org/10 .1126/science.1100907, PubMed: 15486304

Monterosso, J. R., Ainslie, G., Xu, J., Cordova, X., Domier, C. P., \& London, E. D. (2007). Frontoparietal cortical activity of methamphetamine-dependent and comparison subjects performing a delay discounting task. Human Brain Mapping, 28, 383-393. https://doi.org/10.1002/hbm.20281, PubMed: 16944492 
Monterosso, J. R., \& Luo, S. (2010). An argument against dual valuation system competition: Cognitive capacities supporting future orientation mediate rather than compete with visceral motivations. Journal of Neuroscience,

Psychology, and Economics, 3, 1-14. https://doi.org/10.1037 /a0016827, PubMed: 21909453

Nigbur, R., Ivanova, G., \& Stürmer, B. (2011). Theta power as a marker for cognitive interference. Clinical Neurophysiology, 122, 2185-2194. https://doi.org/10.1016/j.clinph.2011.03.030, PubMed: 21550845

O'Hora, D., Carey, R., Kervick, A., Crowley, D., \& Dabrowski, M. (2016). Decisions in motion: Decision dynamics during intertemporal choice reflect subjective evaluation of delayed rewards. Scientific Reports, 6, 20740. https://doi.org/10.1038 /srep20740, PubMed: 26867497

Pinner, J. F. L., \& Cavanagh, J. F. (2017). Frontal theta accounts for individual differences in the cost of conflict on decision making. Brain Research, 1672, 73-80. https://doi.org/10.1016 /j.brainres.2017.07.026, PubMed: 28778686

Pisauro, M. A., Fouragnan, E., Retzler, C., \& Philiastides, M. G. (2017). Neural correlates of evidence accumulation during value-based decisions revealed via simultaneous EEG-fMRI. Nature Communications, 8, 15808. https://doi.org/10.1038 /ncomms15808, PubMed: 28598432

Rangel, A., Camerer, C., \& Montague, P. R. (2008). A framework for studying the neurobiology of value-based decision making. Nature Reviews Neuroscience, 9, 545-556. https:// doi.org/10.1038/nrn2357, PubMed: 18545266

Rodriguez, C. A., Turner, B. M., \& McClure, S. M. (2014). Intertemporal choice as discounted value accumulation. PLOS One, 9, e90138. https://doi.org/10.1371/journal.pone .0090138, PubMed: 24587243

Rosen, B. Q., Padovan, N., \& Marinkovic, K. (2016). Alcohol hits you when it is hard: Intoxication, task difficulty, and theta brain oscillations. Alcoholism: Clinical and Experimental Research, 40, 743-752. https://doi.org/10.1111/acer.13014, PubMed: 27012442

Rustichini, A., \& Padoa-Schioppa, C. (2015). A neurocomputational model of economic decisions. Journal of Neurophysiology, 114, 1382-1398. https://doi.org/10.1152/jn .00184.2015, PubMed: 26063776

Scherbaum, S., \& Dshemuchadse, M. (2020). Psychometrics of the continuous mind: Measuring cognitive sub-processes via mouse tracking. Memory \& Cognition, 48, 436-454. https://doi.org/10.3758/s13421-019-00981-x, PubMed: 31721062

Scherbaum, S., Dshemuchadse, M., Fischer, R., \& Goschke, T. (2010). How decisions evolve: The temporal dynamics of action selection. Cognition, 115, 407-416. https://doi.org/10 .1016/j.cognition.2010.02.004, PubMed: 20227687

Scherbaum, S., Dshemuchadse, M., \& Goschke, T. (2012). Building a bridge into the future: Dynamic connectionist modeling as an integrative tool for research on intertemporal choice. Frontiers in Psychology, 3, 514. https://doi.org/10 .3389/fpsyg.2012.00514, PubMed: 23181048

Scherbaum, S., Dshemuchadse, M., Leiberg, S., \& Goschke, T. (2013). Harder than expected: Increased conflict in clearly disadvantageous delayed choices in a computer game. PLOS One, 8, e79310. https://doi.org/10.1371/journal.pone .0079310, PubMed: 24260192

Scherbaum, S., Frisch, S., \& Dshemuchadse, M. (2018). A bird in the hand isn't good for long: Action dynamics reveal short-term choice impulses in intertemporal choices. Experimental Psychology, 65, 23-31. https://doi.org/10.1027 /1618-3169/a000385, PubMed: 29415644

Scherbaum, S., Frisch, S., Holfert, A.-M., O'Hora, D., \& Dshemuchadse, M. (2018). No evidence for common processes of cognitive control and self-control. Acta
Psychologica, 182, 194-199. https://doi.org/10.1016/j.actpsy .2017.11.018, PubMed: 29202280

Scherbaum, S., Frisch, S., Leiberg, S., Lade, S. J., Goschke, T., \& Dshemuchadse, M. (2016). Process dynamics in delay discounting decisions: An attractor dynamics approach. Judgement and Decision Making, 11, 472-495.

Schoemann, M., \& Scherbaum, S. (2020). From high- to one-dimensional dynamics of decision making: Testing simplifications in attractor models. Cognitive Processing, 21, 303-313. https://doi.org/10.1007/s10339-020-00953-z, PubMed: 32016686

Senftleben, U., Schoemann, M., Rudolf, M., \& Scherbaum, S. (2021). To stay or not to stay: The stability of choice perseveration in value-based decision making. Quarterly Journal of Experimental Psychology, 74, 199-217. https://doi .org/10.1177/1747021820964330, PubMed: 32976065

Senftleben, U., Schoemann, M., Schwenke, D., Richter, S., Dshemuchadse, M., \& Scherbaum, S. (2019). Choice perseveration in value-based decision making: The impact of inter-trial interval and mood. Acta Psychologica, 198, 102876. https://doi.org/10.1016/j.actpsy.2019.102876, PubMed: 31280037

Servan-Schreiber, D., Printz, H., \& Cohen, J. D. (1990). A network model of catecholamine effects: Gain, signal-tonoise ratio, and behavior. Science, 249, 892-895. https://doi .org/10.1126/science.2392679, PubMed: 2392679

Shenhav, A., Botvinick, M. M., \& Cohen, J. D. (2013). The expected value of control: An integrative theory of anterior cingulate cortex function. Neuron, 79, 217-240. https://doi .org/10.1016/j.neuron.2013.07.007, PubMed: 23889930

Shenhav, A., \& Buckner, R. L. (2014). Neural correlates of dueling affective reactions to win-win choices. Proceedings of the National Academy of Sciences, U.S.A., 111, 10978-10983. https://doi.org/10.1073/pnas.1405725111, PubMed: 25024178

Shenhav, A., Straccia, M. A., Cohen, J. D., \& Botvinick, M. M. (2014). Anterior cingulate engagement in a foraging context reflects choice difficulty, not foraging value. Nature Neuroscience, 17, 1249-1254. https://doi.org/10.1038/nn .3771, PubMed: 25064851

Simon, J. R., \& Rudell, A. P. (1967). Auditory S-R compatibility: The effect of an irrelevant cue on information processing. Journal of Applied Psychology, 51, 300-304. https://doi.org /10.1037/h0020586, PubMed: 6045637

Soetens, E., Boer, L. C., \& Hueting, J. E. (1985). Expectancy or automatic facilitation? Separating sequential effects in twochoice reaction time. Journal of Experimental Psychology: Human Perception and Performance, 11, 598-616. https:// doi.org/10.1037/0096-1523.11.5.598

Stroop, J. R. (1935). Studies of interference in serial verbal reactions. Journal of Experimental Psychology, 18, 643-662. https://doi.org/10.1037/h0054651

Töllner, T., Wang, Y., Makeig, S., Müller, H. J., Jung, T.-P., \& Gramann, K. (2017). Two independent frontal midline theta oscillations during conflict detection and adaptation in a Simon-type manual reaching task. Journal of Neuroscience, 37, 2504-2515. https://doi.org/10.1523/JNEUROSCI.1752-16 2017, PubMed: 28137968

Tort, A. B. L., Komorowski, R. W., Manns, J. R., Kopell, N. J., \& Eichenbaum, H. (2009). Theta-gamma coupling increases during the learning of item-context associations. Proceedings of the National Academy of Sciences, U.S.A., 106, 20942-20947. https://doi.org/10.1073/pnas.0911331106, PubMed: 19934062

Usher, M., \& McClelland, J. L. (2001). The time course of perceptual choice: The leaky, competing accumulator model. Psychological Review, 108, 550-592. https://doi.org/10.1037 /0033-295X.108.3.550, PubMed: 11488378 
Verguts, T. (2017). Binding by random bursts: A computational model of cognitive control. Journal of Cognitive

Neuroscience, 29, 1103-1118. https://doi.org/10.1162/jocn a 01117, PubMed: 28253078

Vincent, B. T., \& Stewart, N. (2020). The case of muddled units in temporal discounting. Cognition, 198, 104203. https://doi .org/10.1016/j.cognition.2020.104203, PubMed: 32007801

Voloh, B., Valiante, T. A., Everling, S., \& Womelsdorf, T. (2015). Theta-gamma coordination between anterior cingulate and prefrontal cortex indexes correct attention shifts. Proceedings of the National Academy of Sciences, U.S.A., 112, 8457-8462. https://doi.org/10.1073/pnas.1500438112, PubMed: 26100868

Womelsdorf, T., Vinck, M., Leung, L. S., \& Everling, S. (2010). Selective theta-synchronization of choice-relevant information subserves goal-directed behavior. Frontiers in Human Neuroscience, 4, 210. https://doi.org/10.3389/fnhum .2010.00210, PubMed: 21119780

Yeung, N., Botvinick, M. M., \& Cohen, J. D. (2004). The neural basis of error detection: Conflict monitoring and the error- related negativity. Psychological Review, 111, 931-959. https://doi.org/10.1037/0033-295X.111.4.939, PubMed 15482068

Zauberman, G., Kim, B. K., Malkoc, S. A., \& Bettman, J. R. (2009). Discounting time and time discounting: Subjective time perception and intertemporal preferences. Journal of Marketing Research, 46, 543-556. https://doi.org/10.1509 jmkr.46.4.543

Zavala, B., Jang, A., Trotta, M., Lungu, C. I., Brown, P., \& Zaghloul, K. A. (2018). Cognitive control involves theta power within trials and beta power across trials in the prefrontalsubthalamic network. Brain, 141, 3361-3376. https://doi.org /10.1093/brain/awy266, PubMed: 30358821

Zhao, J., Liang, W.-K., Juan, C.-H., Wang, L., Wang, S., \& Zhu, Z. (2015). Dissociated stimulus and response conflict effect in the Stroop task: Evidence from evoked brain potentials and brain oscillations. Biological Psychology, 104, 130-138. https://doi.org/10.1016/j.biopsycho.2014.12.001, PubMed: 25511611 